\title{
Novel Dominant Rhodopsin Mutation Triggers Two Mechanisms of Retinal Degeneration and Photoreceptor Desensitization
}

\author{
Roustem Iakhine, ${ }^{1 \star}$ Irit Chorna-Ornan, ${ }^{2,4 \star}$ Troy Zars, ${ }^{1}$ Natalie Elia, ${ }^{3,4}$ Yan Cheng, ${ }^{1}$ Zvi Selinger,, 3 Baruch Minke, ${ }^{2,4}$ and \\ David R. Hyde ${ }^{1,5}$ \\ ${ }^{1}$ Department of Biological Sciences, University of Notre Dame, Notre Dame, Indiana 46556, and Departments of ${ }^{2}$ Physiology and ${ }^{3}$ Biological Chemistry and \\ ${ }^{4}$ Kühne Minerva Center for Studies of Visual Transduction, The Hebrew University, Jerusalem, Israel 91120
}

\begin{abstract}
A variety of rod opsin mutations result in autosomal dominant retinitis pigmentosa and congenital night blindness in humans. One subset of these mutations encodes constitutively active forms of the rod opsin protein. Some of these dominant rod opsin mutant proteins, which desensitize transgenic Xenopus rods, provide an animal model for congenital night blindness. In a genetic screen to identify retinal degeneration mutants in Drosophila, we identified a dominant mutation in the ninaE gene $\left(\right.$ NinaE $\left.^{p p 100}\right)$ that encodes the rhodopsin that is expressed in photoreceptors R1-R6. Deep pseudopupil analysis and histology showed that the degeneration was attributable to a light-independent apoptosis. Whole-cell recordings revealed that the NinaE ${ }^{p 100}$ mutant photoreceptor cells were strongly desensitized, which partially masked their constitutive activity. This desensitization primarily resulted from both the persistent binding of arrestin (ARR2) to the NINAE ${ }^{\mathrm{pp} 100}$ mutant opsin and the constitutive activity of the phototransduction cascade. Whereas mutations in several Drosophila genes other than ninaE were shown to induce photoreceptor cell apoptosis by stabilizing a rhodopsinarrestin complex, $N_{i n a} E^{p p 100}$ represented the first rhodopsin mutation that stabilized this protein complex. Additionally, the NinaE $E^{p p 100}$ mutation led to elevated levels of $\mathrm{G}_{\mathrm{q}} \alpha$ in the cytosol, which mediated a novel retinal degeneration pathway. Eliminating both $\mathrm{G}_{\mathrm{q}} \alpha$ and arrestin completely rescued the NinaE ${ }^{p p 100}$-dependent photoreceptor cell death, which indicated that the degeneration is entirely dependent on both $\mathrm{G}_{\mathrm{q}} \alpha$ and arrestin. Such a combination of multiple pathological pathways resulting from a single mutation may underlie several dominant retinal diseases in humans.
\end{abstract}

Key words: Drosophila; desensitization; photoreceptor; rhodopsin; dominant degeneration; arrestin; mutant

\section{Introduction}

Retinitis pigmentosa (RP) is a group of genetic disorders that exhibits abnormal electroretinograms and gradual vision loss attributable to the death of the rod and then cone photoreceptors in humans (Rattner et al., 1999; Phelan and Bok, 2000). Several $\mathrm{X}$-linked and autosomal dominant and recessive forms of RP have been identified (Wang et al., 2001), with rhodopsin mutations being a prevalent cause of autosomal dominant RP (Kaushal and Khorana, 1994; Gal et al., 1997). Rhodopsin is a seven transmembrane, G-protein-coupled receptor that is activated by a photon of light to initiate the phototransduction cascade. Various models describe dominant rhodopsin-mediated

Received Dec. 9, 2003; revised Jan. 22, 2004; accepted Jan. 23, 2004

This work was supported by National Eye Institute Grants R01-EY12426 (D.R.H.) and R01-EY 03529 (B.M., Z.S.), a United States-Israel Binational Science Foundation grant (B.M., D.R.H.), and Israel Science Foundation grants (B.M., Z.S.). We thank Dr. Alexander Kiselev and Dr. Patrick Dolph for sharing their anti-ARR2 antisera and Dr. Joseph $0^{\prime}$ Tousa for kindly providing the ninaE $E^{D 1}$ Drosophila mutant. We also thank Shahar Frechter for providing unpublished data.

*R.I. and I.C.-O. contributed equally to this work.

Correspondence should be addressed to David R. Hyde, Department of Biological Sciences, Galvin Life Science Building, University of Notre Dame, Notre Dame, IN 46556-0369. E-mail: dhyde@nd.edu.

DOI:10.1523/JNEUROSCI.5426-03.2004

Copyright $\odot 2004$ Society for Neuroscience $\quad$ 0270-6474/04/242516-11\$15.00/0 degeneration mechanisms, including the following: (1) defective maturation and/or trafficking of rhodopsin (Roof et al., 1994; Sung et al., 1994), (2) constitutively active rhodopsin and persistent stimulation of the phototransduction cascade (Robinson et al., 1992; Dryja et al., 1993; Rao et al., 1994), and (3) abnormal interactions between rhodopsin and the phototransduction components (Min et al., 1993; Li et al., 1995; Rim and Oprian, 1995).

The Drosophila ninaE gene encodes the rhodopsin protein (NINAE) that is expressed in six (R1-R6) of the eight adult photoreceptor cells in each ommatidium (O'Tousa et al., 1985; Zuker et al., 1985). The NINAE protein is activated by blue light, which stimulates the $d g q$-encoded $\mathrm{G}_{\mathrm{q}} \alpha$ subunit of the heterotrimeric G-protein (Lee et al., 1990, 1994; Scott et al., 1995). The photoactivated metarhodopsin is inactivated by phosphorylation and arrestin (ARR2) binding (Byk et al., 1993; Dolph et al., 1993; Kiselev and Subramaniam, 1994; Alloway and Dolph, 1999). Orange light photoconverts metarhodopsin to rhodopsin, which dissociates from arrestin and is dephosphorylated by the $r d g C$ encoded $\mathrm{Ca}^{2+}$-dependent serine/threonine phosphatase (Byk et al., 1993). In the absence of arrestin dissociation, the persistent NINAE-arrestin complexes are endocytosed and induce apoptosis of photoreceptor cells (Alloway et al., 2000; Kiselev et al., 2000). Recessive ninaE mutants exhibit a defective light response 
and photoreceptor cell death (Leonard et al., 1992). Dominant ninaE mutations were identified as suppressors of either $r d g C$ mediated (Kurada and O'Tousa, 1995) or $r d g B$-dependent (Colley et al., 1995) retinal degeneration. The $r d g B$ gene encodes a novel phosphatidylinositol transfer protein that is required for the light response and photoreceptor viability (Vihtelic et al., 1991, 1993). All of the previously isolated dominant ninaE mutants are defective in the maturation and/or trafficking of rhodopsin in the endoplasmic reticulum (ER) (Colley et al., 1995; Kurada et al., 1998). Thus, dominant ninaE mutations likely mimic the degeneration mechanisms underlying rhodopsinmediated autosomal dominant RP in humans.

We report a new dominant ninaE mutant $\left(N_{i n a E^{p p 100}}\right)$ that exhibits retinal degeneration and constitutively active and strongly desensitized photoreceptor cells. The photoreceptor cell desensitization and death resulted from both the NINAE ${ }^{\text {pp } 100}$ mutant rhodopsin forming a stable complex with arrestin and the persistent localization of the $\mathrm{G}_{\mathrm{q}} \alpha$ in the cytosol. Genetic experiments reveal that the arrestin and $\mathrm{G}_{\mathrm{q}} \alpha$ mechanisms are mutually exclusive. Furthermore, the predominant localization of an active $\mathrm{G}_{\mathrm{q}} \alpha$ protein in the cytosol stimulates photoreceptor cell death through a novel mechanism.

\section{Materials and Methods}

Isolation of the dominant NinaE $E^{p 100}$ mutant. Wild-type males were starved for $6 \mathrm{hr}$ and then fed $25 \mathrm{~mm}$ ethyl methanesulfonate (Sigma, St. Louis, MO) in a $1 \%$ sucrose solution overnight (Lewis and Bacher, 1968). The males were mated en masse to $S M 1 / S c o$; TM2/Sb virgin females, and the F1 progeny were raised in constant light for $10 \mathrm{~d}$. The F1 flies were screened for the absence of a wild-type deep pseudopupil, which is a virtual image of the rhabdomeres from several adjacent ommatidia (Franceschini, 1972).

Characterizing the retinal degeneration phenotype. Flies were raised under constant light and scored daily for the presence of the deep pseudopupil as described previously (Paetkau et al., 1999). The average percentage of flies possessing a wild-type deep pseudopupil and SDs were determined from at least four replicates (100-150 flies per trial) of each genotype and age. Flies raised in constant darkness were collected, aged until a designated day, scored for the presence or absence of a wild-type deep pseudopupil, and then discarded.

For light and electron microscopy of retinal sections, flies were raised in a $12 \mathrm{hr}$ light/dark cycle to the desired age and then decapitated. The heads were bisected, fixed, and embedded in Polybed 812 as described previously (Lee et al., 1994). Sections for light and electron microscopy were generated and processed as described previously (Paetkau et al., 1999).

DNA isolation and sequencing. Genomic DNAs from wild-type and NinaE $E^{p p 100}$ mutant flies were isolated as described previously (Ashburner, 1989). Wild-type and mutant ninaE genes were PCR amplified using the 5' AGG ATC CAA TGG AGA GGT ACG ATC GGT GAA TCC AC and 5' GGT TGT GGA TCC AAA GAA TTT ATG CC primers that mapped to nucleotides -8 and 1518 of the ninaE gene, respectively (O'Tousa et al., 1985). Both PCR primers contain engineered BamHI sites at their $5^{\prime}$ ends (in bold), and the first primer also deletes a BamHI site (underlined) in the first ninaE intron. The PCR reactions contained $3 \mu \mathrm{g}$ of genomic DNA, $1 \times$ native $P f u$ buffer, $2.5 \mathrm{~mm}$ of each dNTP, and $250 \mathrm{pmol}$ of each primer. After incubating at $96^{\circ} \mathrm{C}$ for $7 \mathrm{~min}, 1 \mu \mathrm{l}$ of native Pfu DNA polymerase (Stratagene, La Jolla, CA) was added and amplified through 30 cycles of $96^{\circ} \mathrm{C}$ for $1 \mathrm{~min}, 54^{\circ} \mathrm{C}$ for $1 \mathrm{~min}$, and $72^{\circ} \mathrm{C}$ for $4 \mathrm{~min}$, followed by a $10 \mathrm{~min}$ extension at $72^{\circ} \mathrm{C}$. The PCR products were size fractionated on a Tris acetate-EDTA agarose gel, purified with the Wizard PCR prep resin (Promega, Madison, WI), and blunt-end ligated into the pZero 2.1 vector (Invitrogen, Carlsbad, CA). The plasmid DNA was purified with Strataclean Resin (Stratagene), precipitated, resuspended in $\mathrm{H}_{2} \mathrm{O}$, and sequenced using the Sequenase Quick Denature Kit (US Biochemicals, Cleveland, $\mathrm{OH}$ ), with primers generated from the published ninaE genomic sequence (O’Tousa et al., 1985).
In vitro mutagenesis. The $P f u$-generated wild-type ninaE gene was digested from pZero 2.1 with $B a m H I$, gel purified, and cloned into the BamH1 site of the PK- ATG - vector, which contains the $3.2 \mathrm{~kb}$ ninaE promoter and a $0.7 \mathrm{~kb}$ ninaE poly $\left(\mathrm{A}^{+}\right)$tail (Kurada and O'Tousa, 1995; Shetty et al., 1998). The NinaE ${ }^{p 100}$ mutation was introduced into the wild-type ninaE gene by in vitro mutagenesis in the PK-ATG - vector using the Quick Change Site-Directed Mutagenesis kit (Stratagene) and the complementary primer pairs 5' TCA ACT GCA TGA GAC TGT TCA AGT $3^{\prime}$ and 5' ACT TGA ACA GTC TCA TGC AGT TGA 3' (the site of the nucleotide change is in bold). The PCR reaction contained $50 \mathrm{ng}$ of template DNA, $125 \mathrm{ng}$ of each primer, $1 \times$ buffer, $10 \mathrm{~mm}$ of each dNTP, and $1 \mu \mathrm{l}$ of $P f u$ DNA polymerase. The PCR amplification protocol was $95^{\circ} \mathrm{C}$ for $30 \mathrm{sec}$ and then $12 \mathrm{cycles}$ of $95^{\circ} \mathrm{C}$ for $30 \mathrm{sec}, 60^{\circ} \mathrm{C}$ for $1 \mathrm{~min}, 68^{\circ} \mathrm{C}$ for $2 \mathrm{~min}$, and a final $15 \mathrm{~min}$ extension at $68^{\circ} \mathrm{C}$.

Germ-line transformation. A $5.4 \mathrm{~kb} K p n \mathrm{I}$ fragment containing the ni$n a E$ promoter, the in vitro mutagenized ninaE genomic DNA, and the ninaE poly $\left(\mathrm{A}^{+}\right)$tail was gel purified and cloned into the KpnI site of the pCaSpeR-4 transformation vector (Ashburner, 1989). The sequence of the entire ninaE coding region was confirmed in this vector. This in vitro mutagenized DNA was germ-line transformed with the $\Delta 2-3$ P-element (ratio of 6:1) using standard techniques (Spradling, 1986).

Electrophysiology. Electroretinograms (ERGs) were performed as described previously (Paetkau et al., 1999). ERG traces were recorded using a MacAdios II analog-to-digital converter using SuperScope II software on a Macintosh IIx computer (Apple Computers, Cupertino, CA).

To measure light-induced currents (LICs), orange light (OG 590 Ditric edge filter) from a xenon high-pressure lamp $(75 \mathrm{~W})$ was delivered to isolated ommatidia via the objective lens ( $60 \times$ Olympus Optical, Tokyo, Japan) and attenuated up to seven orders of magnitude by neutral density filters. The maximal luminous intensity of the orange light at the level of the ommatidia was $\sim 3.0 \log$ units above the intensity required for a half-maximal response of the R1-R6 photoreceptors. The effective intensity of the white light without the orange filter was determined by comparing the light intensities with and without the orange filter, which were required to elicit a small response of the same amplitude in wild-type flies. In Figure 4, the intensity of the white light stimulus was converted to the effective relative intensity of orange light.

Dissociated ommatidia were prepared from either newly eclosed adult flies ( $<1 \mathrm{hr}$ after eclosion) or late pupae as described previously (Hardie, 1991). Whole-cell patch-clamp recordings were performed as described previously (Hardie and Minke, 1992; Peretz et al., 1994). Recordings were made at $21^{\circ} \mathrm{C}$ using patch pipettes of 5-10 $\mathrm{M} \Omega$ pulled from fiberfilled borosilicate glass capillaries. Series resistance of 7-14 $\mathrm{M} \Omega$ was carefully compensated $(>80 \%)$ during all experiments. Signals were amplified with an Axopatch-1D or 2B (Axon Instruments, Foster City, CA) patch-clamp amplifier, sampled at $2 \mathrm{kHz}$, and filtered below $1 \mathrm{kHz}$.

Immunoblots of NINAE, ARR2, and $G_{q} \alpha$ proteins. Immunoblots of rhodopsin expression in flies 4-6 hr old were performed as described previously (Milligan et al., 1997). Head protein homogenates were electrophoresed, electrotransferred to Hybond polyvinylidene difluoride (PVDF) membrane (Amersham Biosciences, Piscataway, NJ), and incubated with the 4C5 anti-rhodopsin monoclonal antibody (Developmental Studies Hybridoma Bank, University of Iowa, Iowa City, IA). The 4C5 monoclonal antibody was detected with HRP-conjugated goat antimouse antibodies (1:5000) and ECL reagents (Amersham Biosciences). Band intensity was quantified on a scanning laser densitometer (BioRad, Hercules, CA) and normalized to a standard curve generated by a dilution series of head protein extract, with five independent blots analyzed.

The ARR2 protein was isolated under dim red light from white-eyed wild-type and mutant fly heads that were $4 \mathrm{hr}$ after eclosion. The heads were placed in $20 \mu$ l of PBS, pH 7.4, illuminated at the desired wavelength for $2 \mathrm{~min}$, homogenized in darkness, and centrifuged with $15,000 \times g$. The supernatant fraction was transferred to a new Eppendorf tube (Eppendorf Scientific, Westbury, NY), and the pellet was resuspended in 20 $\mu \mathrm{l}$ of PBS. SDS extraction buffer $(20 \mu \mathrm{l})$ was added to both fractions and incubated at $45^{\circ} \mathrm{C}$ for $1 \mathrm{hr}$ before electrophoresing one head equivalent of cytosolic and membrane protein extracts through a $7-15 \%$ gradient SDS-PAGE gel. After electrotransfer, the PVDF membranes were incu- 
bated with a mixture of the $4 \mathrm{C} 5$ anti-rhodopsin monoclonal antibody and anti-ARR2 rabbit polyclonal antisera (Alloway et al., 2000; Kiselev et al., 2000) and detected with a mixture of HRP-conjugated mouse anti-rabbit and rabbit anti-mouse antibodies and the ECL system (Amersham Biosciences), with five independent blots analyzed.

The $\mathrm{G}_{\mathrm{q}} \alpha$-protein was quantitated in different subcellular fractions as described previously (Kosloff et al., 2003). Wild-type and NinaE $E^{\text {pp100 }}$ flies were dark adapted for $24 \mathrm{hr}$ and then subjected to either blue light illumination $(490 \mathrm{~nm})$ or continued darkness for $10 \mathrm{~min}$ at $22^{\circ} \mathrm{C}$. The heads were homogenized at $4^{\circ} \mathrm{C}$ in the dark in hypotonic medium (20 mM HEPES buffer, $\mathrm{pH}$ 7.6, containing $15 \mathrm{~mm} N$-ethylmaleimide, 20 $\mu \mathrm{g} / \mathrm{ml}$ leupeptin, $1 \mu \mathrm{g} / \mathrm{ml}$ pepstatin $\mathrm{A}$, and 0.35 $\mathrm{mg} / \mathrm{ml} o$-phenantroline). The samples were centrifuged at $15,800 \times g$ for $15 \mathrm{~min}$ at $4^{\circ} \mathrm{C}$ to separate the membrane pellet from the soluble proteins. The pellet was washed and centrifuged, and the supernatants were combined. Ultracentrifugation at $150,000 \times g$ for $30 \mathrm{~min}$ did not change the $\mathrm{G}_{\mathrm{q}} \alpha$ distribution between the fractions. The proteins were precipitated in $5 \%$ TCA, electrophoresed on a $10 \%$ SDSPAGE, and electrotransferred to nitrocellulose using a semidry transfer apparatus (Bio-Rad). The nitrocellulose was blocked and incubated with a rabbit anti- $\mathrm{G}_{\mathrm{q}} \alpha$ polyclonal antibody raised against the decapeptide of the $\mathrm{C}$ terminus of the protein. The nitrocellulose was incubated with anti-rabbit polyclonal antiserum and detected with ECL Amersham Biosciences reagents according to the instructions of the manufacturer and quantitated with Fuji LAS-1000. The amount of $\mathrm{G}_{\mathrm{q}} \alpha$ in each fraction was calculated as a percentage of the total $\mathrm{G}_{\mathrm{q}} \alpha$ in the pellet and supernatant, with four independent blots analyzed.

GTPase assay. GTP hydrolysis of Drosophila head extracts under various light conditions were performed essentially as described previously (Blumenfeld et al., 1985).

\section{Results}

The NinaE ${ }^{p p 100}$ mutant exhibits a dominant retinal degeneration and a recessive electrophysiological defect

In a screen to identify Drosophila mutants that lacked a deep pseudopupil, we identified a dominant retinal degeneration mutation that mapped $<1 \mathrm{cM}$ from the ninaE gene, which encodes the rhodopsin protein expressed in photoreceptor cells R1-R6 (O’Tousa et al., 1985; Zuker et al., 1985). To determine whether NinaE $E^{p 100}$ was a ninaE allele, we examined the ERGs of flies that were $\sim 12 \mathrm{hr}$ after eclosion (before any signs of degeneration). White-eyed wild-type flies exhibited a prolonged depolarizing afterpotential (PDA) after a blue light converts a large fraction of rhodopsin to metarhodopsin (Fig. 1A) (Hillman et al., 1983; Dolph et al., 1993). The white-eyed nina $E^{I 17} /$ ninaE $E^{I 17}$ null mutant lacked a PDA and possessed small light response amplitudes (Fig. $1 B$ ), which were attributable to the opsins expressed in photoreceptor cells R7 and R8. White-eyed NinaE $E^{p 100} /$ ninaE $^{+}$flies exhibited a wild-type ERG light response and a PDA (Fig. 1C), whereas NinaE $E^{p 100} / N_{i n a E} E^{p 100}$ flies lacked the PDA (Fig. 1E). Thus, the NinaE $E^{p 100}$ flies exhibited a recessive mutant ERG phenotype. White-eyed NinaE $E^{p 100} /$ ninaE $E^{I 17}$ flies also lacked the PDA (Fig. $1 D)$, which confirmed that NinaE $E^{p 100}$ was a ninaE allele.
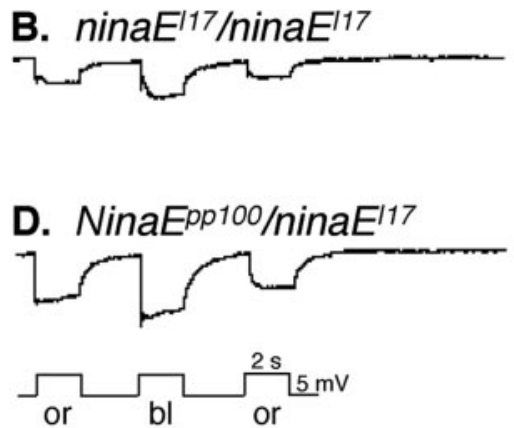

Figure 1. NinaE $E^{p p 100}$ is a ninaE allele. We recorded the ERG light response of wild-type $(A)$, ninaE $^{[17} /$ ninaE $^{[17}(B)$, Nina $E^{p p 100} /$ with orange light (or), 2 sec with blue light (bl), and a final 2 sec with orange light, as shown below $D$. Wild-type flies exhibited a PDA in response to blue light $(A, C$, which was absent in ninaE mutant flies $(B, D, E)$. Calibration for $E R G$ responses is shown below 政 $\mathrm{EE}^{117}$ flies $(12 \% \pm 3.1 \%)$. G, The putative amino acid sequence of the NINAE opsin protein is shown as a single letter amino acid code. The $\mathrm{N}$ and $\mathrm{C}$ termini are labeled $\left(\mathrm{NH}_{2}\right.$ and $\mathrm{COOH}$, respectively), with the relative orientation of the intracellular and extracellular faces of the membrane labeled. The NINAE ${ }^{\text {pp100 }}$ mutation (G299R) is shown.

Immunoblots revealed that the NinaE ${ }^{p 100}$ mutant gene expressed $\sim 20 \%$ of the rhodopsin relative to the nina $E^{+}$gene (Fig. $1 F)$. We sequenced the ninaE gene from the NinaE $E^{p p 100}$ mutant and identified a G-to-A transition mutation, which changed the glycine at amino acid 299 to arginine (Fig. 1G).

We examined the retinal degeneration that underlies the deep pseudopupil loss in the NinaE $E^{p 100}$ mutant. A 2-d-old wild-type retina possessed a regular repeating pattern of ommatidia (Fig. $2 \mathrm{~A}$, left column), whereas the ultrastructure of a 10 -d-old retina revealed large R1-R6 rhabdomeres surrounding the central R7 rhabdomere (Fig. $2 A$, right column). In contrast, the 2-d-old $\mathrm{NinaE}^{\mathrm{pp} 100} /$ ninaE $^{+}$retina possessed a few holes and reduced or missing rhabdomeres (Fig. 2 B). Electron microscopy of the 10d-old NinaE $\mathrm{Pp}^{\mathrm{p} 100} /$ ninaE $^{+}$retina revealed degenerating R1-R6 photoreceptors (darkly stained) and phagocytosed photoreceptors (Fig. $2 \mathrm{~B}$, asterisks). To prove that the G299R mutation caused the dominant NinaE $E^{p 100}$ mutant phenotype, we created three independent transgenic lines that contained a wild-type ninaE gene that was in vitro mutagenized to introduce the G299R mutation. At both the light and electron microscope level, all three transgenic lines (ninaE ${ }^{+}, P\left[\right.$ ninaE-G299R]/ninaE ${ }^{I 17}$ ) (Fig. $2 C$ ) exhibited a dominant degeneration phenotype that was indistinguishable from the NinaE $E^{p 100} /$ ninaE $^{+}$flies. Therefore, the G299R mutation accounted for the dominant NinaE ${ }^{p 100}$ degeneration phenotype.

Because the NinaE $E^{p 100}$ mutant exhibited a recessive ERG light 

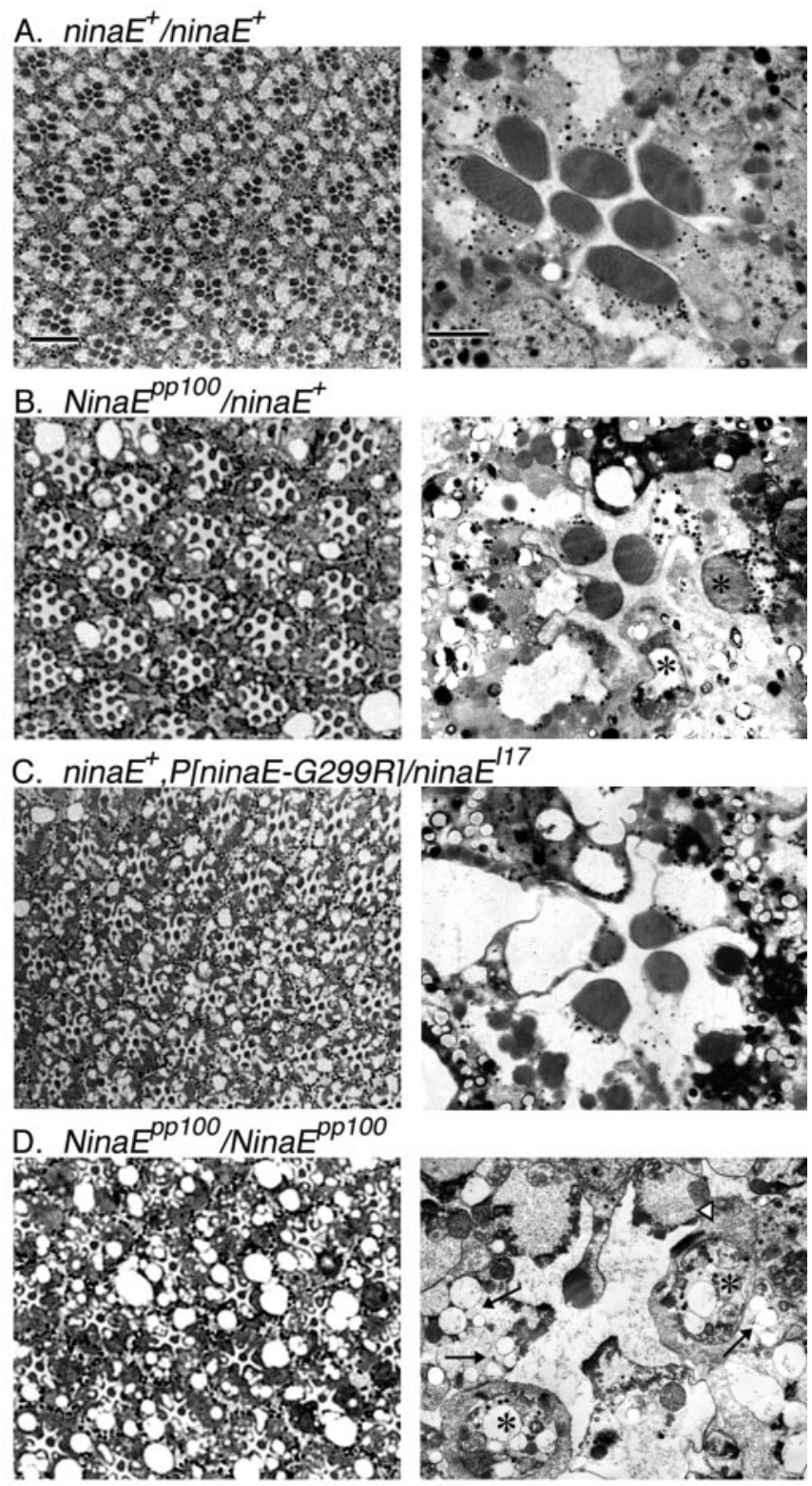

Figure 2. The Nina $E^{p p 100}$ retinal degeneration phenotype. The histology of wild-type (ninaE $^{+} /$ninaE $\left.{ }^{+} ; A\right)$, NinaE ${ }^{p p 100} /$ ninaE $^{+}(B)$, ninaE $^{+}, P\left[\right.$ ninaE-G299R]/ninaE ${ }^{177}$ transgenic flies

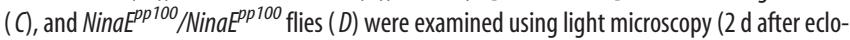
sion; left column) and electron microscopy (10 $\mathrm{d}$ after eclosion; right column). The dominant Nina $E^{p 100}(B)$ degeneration phenotype was characterized by loss of rhabdomeres, presence of intracellular vacuoles, and phagocytosis of photoreceptors (asterisks). The transgenic flies ( $C$ ) exhibited a degeneration that was indistinguishable from the dominant mutant phenotype, which confirmed that the G299R mutation caused the dominant degeneration. The homozygous NinaE ${ }^{\text {pp } 100}$ degeneration $(D)$ was characterized by a rapid loss of the rhabdomeres, increased number of large intracellular vesicles (arrows), phagocytosis of the photoreceptor cells (asterisk), and cytoplasmic condensation (arrowhead). Scale bars (in A): left, $10 \mu \mathrm{m}$; right, 1 $\mu \mathrm{m}$ (shown in the wild-type sections and are the same for the subsequent sections).

response defect, we examined the histology of NinaE $E^{p 100}$ homozygotes. The 2-d-old NinaE $E^{p 100} / N_{i n a E^{p p 100}}$ retina exhibited an increased number of large intracellular vesicles, more ommatidial disorganization, and fewer rhabdomeres relative to Nina$E^{p p 100 / n i n a E^{+}}$(Fig. 2D, B, respectively). The ultrastructure of a 10-d-old NinaE $E^{p 100} /$ NinaE $^{\text {pp100 }}$ ommatidium revealed that the R1-R6 rhabdomeres were more disorganized and smaller than the central R7 rhabdomere, and many large intracellular vesicles (Fig. 2D, arrows) were present within some of the R1-R6 photo-

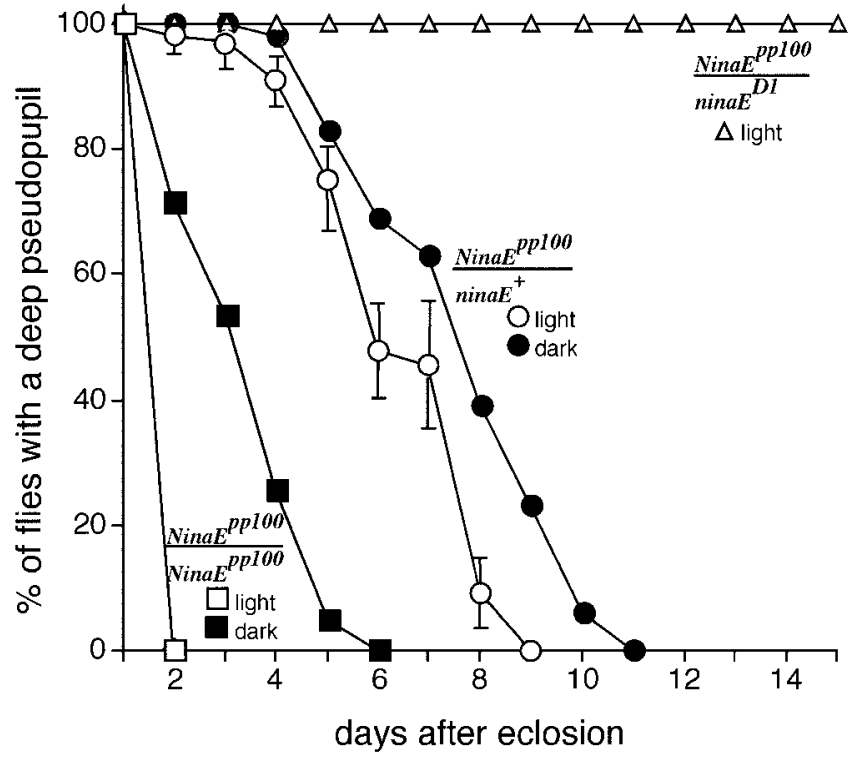

Figure 3. Rates of NinaE ${ }^{p p 100}$ deep pseudopupil loss. We examined the time course of deep pseudopupil loss of NinaE $E^{p 100} /$ NinaE $^{p p 100}$ flies (squares), NinaE $E^{p 100} /$ ninaE $^{+}$flies (circles), and

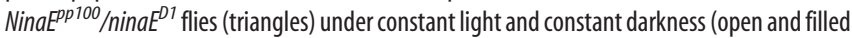
symbols, respectively). The homozygous NinaE $E^{p 100}$ mutants exhibited an earlier and more rapid deep pseudopupil loss relative to the heterozygous NinaE ${ }^{p 100}$ mutants. Furthermore, both the homozygous and heterozygous NinaE $E^{\text {pp } 100}$ mutants exhibited more rapid deep pseudopupil loss in constant light relative to constant darkness. The nina $E^{D 1}$ mutation strongly suppressed the rapid NinaE ${ }^{p 100}$ deep pseudopupil loss (triangles). The percentages of flies retaining a deep pseudopupil on each day were plotted against their age, with each symbol representing the average from four independent trials of 100-150 flies per trial.

receptors. Some photoreceptors were being phagocytosed, and others exhibited a decreased cytoplasmic volume (Fig. $2 \mathrm{D}$, asterisks, arrowhead, respectively). Thus, the NinaE ${ }^{p 100}$ mutation caused a dominant retinal degeneration that was enhanced in the Nina $E^{p p 100}$ homozygote.

The NinaE ${ }^{p p 100}$ mutant exhibits a light-enhanced retinal degeneration

We examined the effect of light on the NinaE $E^{p p^{100}}$ retinal degeneration to differentiate between various potential degeneration mechanisms. We used the deep pseudopupil as a rapid and noninvasive technique to assay the integrity of the retina and photoreceptor cells (Franceschini, 1972). The NinaE ${ }^{p p 100}$ mutant exhibited a dominant deep pseudopupil loss that was slightly faster in constant light than in constant darkness (Fig. 3, NinaE $E^{p p 100}$ / nina $E^{+}$, open circles, filled circles, respectively). The absence of any wild-type $\mathrm{NINAE}^{+}$protein in the $\mathrm{NinaE}^{\mathrm{pp100}} / \mathrm{NinaE}^{\mathrm{pp} 100}$ flies dramatically increased the onset and rate of deep pseudopupil loss relative to $\mathrm{NinaE}^{\mathrm{pp} 100} /$ ninaE $^{+}$(Fig. 3). Furthermore, the Ni$n a E^{p p 100} /$ Nina $^{p p 100}$ degeneration time course exhibited a significantly faster deep pseudopupil loss in constant light relative to constant darkness (Fig. 3, open squares, filled squares, respectively). The NinaE $E^{p 100} /$ ninaE $E^{I 17}$ flies exhibited a deep pseudopupil loss that mimicked the NinaE $E^{p 100} / N_{i n a E} E^{p 100}$ degeneration time course (data not shown). The nina $E^{D 1}$ mutation, which is a serine-to-phenylalanine change at position 137 in the third transmembrane domain, causes a slow dominant retinal degeneration by blocking rhodopsin maturation in the endoplasmic reticulum (Kurada and O'Tousa, 1995; Kurada et al., 1998). The ninaE ${ }^{D 1}$ mutation suppressed the rapid Nina $E^{p 100}$-dependent deep pseudopupil loss (Fig. 3, NinaE $E^{p 100} / n i n a E^{D 1}$, triangles). Therefore, 
the NinaE $E^{p 100}$ mutation must cause its rapid degeneration through a mechanism subsequent to the nina $E^{D 1}$ block of rhodopsin maturation in the ER (Kurada and O'Tousa, 1995).

\section{The NinaE ${ }^{p p 100}$ mutant is strongly desensitized}

We performed whole-cell recordings on the homozygous Nina$E^{p p 100}$ mutant before photoreceptor degeneration to elucidate the underlying physiological defect. The wild-type LIC to dim orange light stimulus showed current fluctuations that are attributable to the summation of the quantum bumps (Fig. 4A, top left panel), whereas a bright orange light stimulus invoked an initial transient phase that declined to a small steady-state phase attributable to light adaptation (Fig. $4 A$, top right panel). The LIC from one of the most sensitive Nina $E^{p p 100}$ mutant cells to intense orange light elicited only a small response that lacked large current fluctuations (Fig. $4 A$, middle left panel). Furthermore, the intense white light required to elicit a sizable response in the Nina $^{p p 100}$ mutant (Fig. $4 A$, middle right panel) was approximately four orders of magnitude brighter than the stimulus eliciting a similar response amplitude in a wild-type cell. The need for a more intense light stimulus to induce a wild-type-sized LIC response in NinaE $E^{p p 100}$ suggested that the NinaE $E^{p 100}$ mutant possessed a highly reduced sensitivity to light. The individual NinaE ${ }^{p p 100}$ cells exhibited a large variability in their sensitivity to light, with only a fraction of the light-sensitive NinaE $E^{p 100}$ mutant cells possessing the maximal peak response amplitude (data not shown). Because this amplitude was similar to that of wild-type flies, the mutant cells must contain a sufficient number of light-sensitive rhodopsin molecules. It was shown previously that the high gain of the phototransduction cascade resulted in the ninaE $E^{P 332}$ mutant exhibiting a shift of $\sim 3.5$ orders of magnitude in the intensity-response function ( $V$-log $I$ curve) and a wild-type peak amplitude of the receptor potential, although it had approximately three orders of magnitude less rhodopsin than wild-type flies (Johnson and Pak, 1986).

To confirm the reduced light sensitivity of the NinaE $E^{p 100}$ cells, we plotted the intensity-response function ( $i$-log $I$ curve) for several different individual NinaE $E^{p p 100}$ mutant and wild-type cells (Fig. $4 B$ ). There was a large difference in the light intensity range at which wild-type and the NinaE ${ }^{p p 100}$ photoreceptors responded, with a considerable fraction of mutant cells either not responding to the maximal white light intensity or only exhibiting minimal response amplitudes. This data clearly demonstrated that a main electrophysiological phenotype of the NinaE $E^{p 100}$ mutant relative to wild type is a large decrease in the sensitivity to light.

\section{The NinaE $E^{p p^{100}}$ desensitization is partially rescued by elimination of arrestin}

One mechanism that could underlie the NinaE $E^{p p 100}$ reduced light sensitivity is persistent binding of arrestin (ARR2) to NINA$\mathrm{E}^{\mathrm{Pp} 100}$. Persistent arrestin binding was shown previously to result in retinal degeneration of several Drosophila mutants (Alloway et al., 2000; Kiselev et al., 2000) and the transgenic mouse model expressing the K296E mutant human opsin (Li et al., 1995). Whole-cell recordings on the arr ${ }^{\text {Y2OSTOP NinaE }}{ }^{\text {pp100 }}$ double mutant (Fig. $4 A$, bottom left panel) revealed the bump noise that was also observed in wild-type flies (Fig. $4 A$, top left panel), but absent in NinaE ${ }^{p p 100}$ (Fig. 4A, middle left panel). Although the arr $2^{Y 20 S T O P}$ NinaE $E^{p p 100}$ double mutant revealed a large increase in light sensitivity relative to $N i n a E^{p p 100}$, it was still significantly less sensitive than wild type (Fig. $4 A$, bottom right panel). We compared the intensity-response function ( $i-\log I$ curve) of the
A. wild type
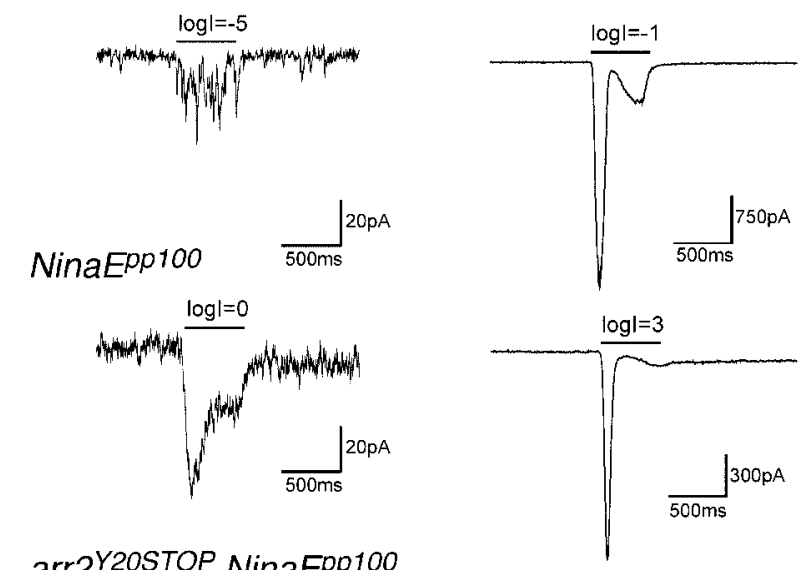

arr2Y20STOP NinaEpp100
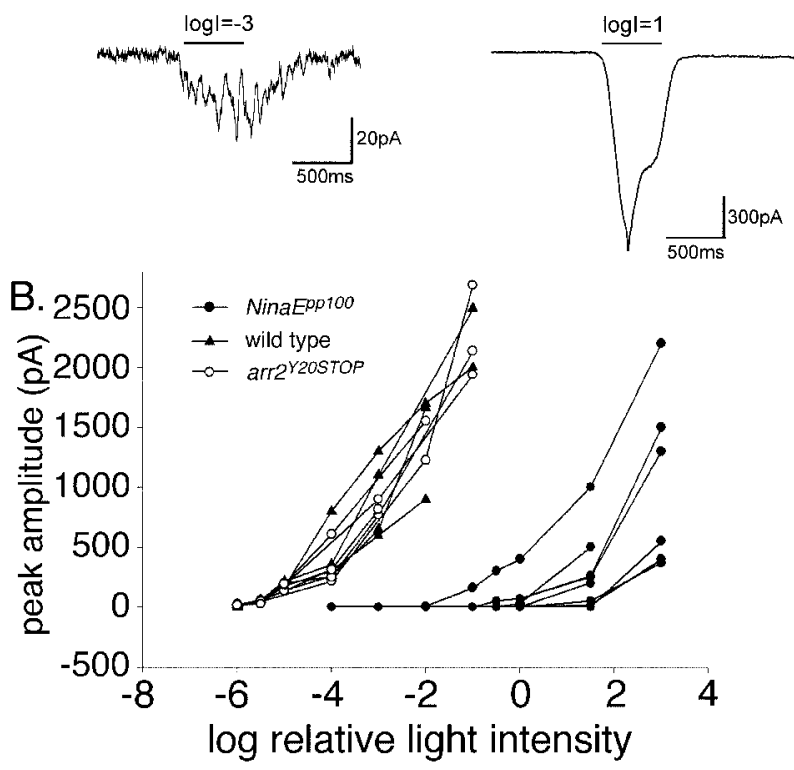

Figure 4. LICS of Nina $E^{p p 100}$ mutants exhibit a highly reduced sensitivity to light relative to wild-type flies. A, Top row, LIC of a wild-type photoreceptor cell in response to $\operatorname{dim}(\log I=$ $-5.0)$ and more intense $(\log I=-1.0)$ orange light pulses indicated by horizontal bars. The response to the dim light shows the typical current fluctuations attributable to the summation of quantum bumps. The response to intense light shows the initial transient phase that declines to a small steady-state phase attributable to light adaptation. Note the different current scales below all of the responses. A, Middle row, LIC of a Nina ${ }^{p p 100}$ photoreceptor cell in response to intense orange light $(\log I=0)$ and more intense white light expressed in equivalent orange light intensity $(\log I=3)$. No bump noise is observed. A, Bottom row, LIC of an arr2 ${ }^{\text {Y2ostoP }}$ Nina $E^{p 100}$ photoreceptor cell in response to medium intensity orange light $(\log I=-3)$ and more intense white light expressed in equivalent orange light intensity $(\log I=1)$. Note the appearance of bump noise at the lower light intensity. The slower LIC waveforms at both light intensities are typical for the arr2 mutant phenotype. B, Families of intensity-response functions ( $\left(i-\log /\right.$ curves) obtained from wild-type, NinaE $E^{\text {pp } 100}$, and arr2 ${ }^{\text {Y2OSTOP }}$ mutant photoreceptors. The graph plots the peak current amplitudes of the responses (in picoamperes) for wildtype (filled triangles), NinaE ${ }^{\text {pp } 100}$ (filled circles), and arr2 ${ }^{\text {Y20sToP }}$ (open circles) mutant photoreceptors. Only a few of the NinaE $E^{p p 100}$ mutant cells responded to the intense orange light stimulus. The light intensity range above $\log I=0$ was obtained with white light that was converted to equivalent orange intensity (see Materials and Methods). The arr2 ${ }^{\text {Y2ostoP }}$ cells possessed $i-\log /$ curves that were indistinguishable from wild type.

arr2 $2^{\text {Y2OSTOP }}$ mutant photoreceptors with wild-type and Nina$E^{p p 100}$ cells and found that the family of $\operatorname{arr} 2^{Y 20 S T O P}$ responses was nearly identical to wild type (Fig. $4 B$ ). Thus, the ARR2 protein specifically interacted with the NINAE ${ }^{\mathrm{Pp} 100}$ mutant rhodopsin to decrease the sensitivity to light. However, this interaction failed 

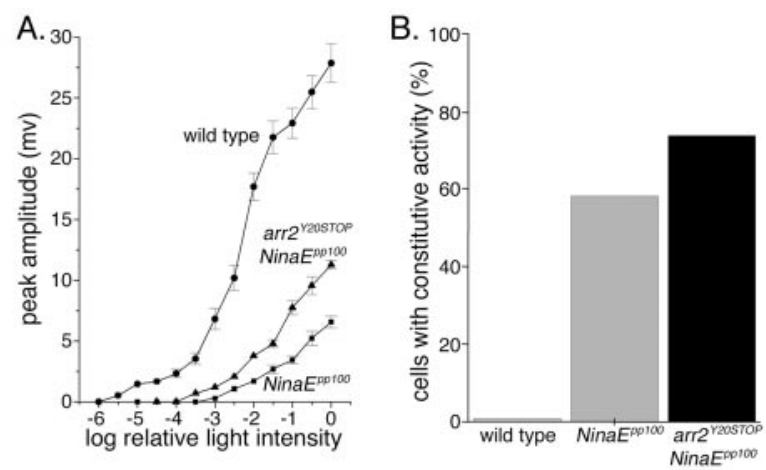

C. wild type
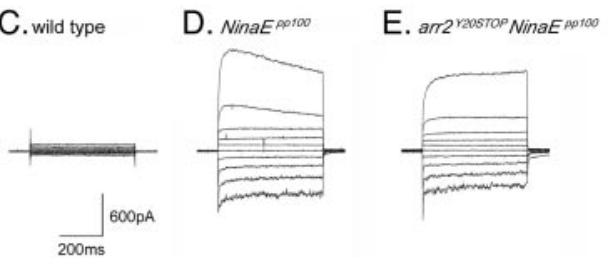

$\mathrm{F}$.

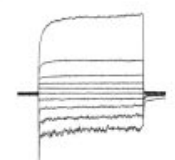

F. arr2 $2_{+\mathrm{Lacos}}$ NinaE Aos 100
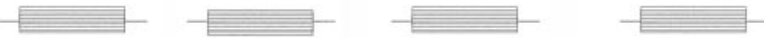

Figure 5. The Nina $\mathrm{E}^{p 100}$ mutation induces constitutive activity of the light-sensitive channels. $A$, In vivo $E R G$ recordings of intensity-response functions measured in wild-type (filled circles; $n=6$ ), NinaE $E^{p p 100}$ (filled squares; $n=8$ ), and $\operatorname{arr}^{Y 20 S T O P}$ NinaE ${ }^{p p 100}$ (filled triangles; $n=6$ ). The arr2 ${ }^{Y 20 S T O P}$ mutation increased the NinaE ${ }^{p p 100}$ sensitivity to light $\sim 10$-fold. $B$, Histograms plotting the percentage of NinaE $E^{p 100}$ ( $n=33$ adult flies and 10 pupae) and $\operatorname{arr}{ }^{\text {Y2OSTOP }} \mathrm{NinaE}^{\mathrm{pp} 100}$ ( $n=23$, all pupae) cells showing constitutive activity in the dark. The $\operatorname{arr} 2^{\text {Y2OSTOP }}$ mutation increased the fraction of NinaE $E^{p p 100}$ cells exhibiting constitutive activity. $(-F$, Families of current traces elicited by a series of $20 \mathrm{mV}$ voltage steps from -100 to $+80 \mathrm{mV}$ (bottom row). A wild-type photoreceptor in the dark exhibits only a small leak current ( $C$ ). Both NinaE $E^{p p 100}$ and $\operatorname{arr}^{Y 20 S T O P}$ NinaE $E^{p p 100}$ pupal photoreceptors possess inward and outward rectifying currents in the dark $\left(D, E\right.$, respectively). Addition of $20 \mu \mathrm{m} \mathrm{La}{ }^{3+}$ to the external medium of the same $\operatorname{arr}^{Y 20 S T O P}$ NinaE ${ }^{p p 100}$ pupal cell almost completely abolished the current $(F)$. Very similar results were obtained when adult (newly eclosed) flies were used, except that, at negative holding potentials, the inward currents were smaller. Calibration is shown under the wild-type traces $(C)$ and is the same for $D-F$.

to account for the entire range of reduced sensitivity of the Nina$E^{p p 100}$ cells.

We extended this analysis by plotting the peak amplitude versus log of the relative light intensity from in vivo ERG recordings and confirmed that the arr2 $2^{Y 20 S T O P} N_{i n a E}{ }^{p p 100}$ double mutant possessed an increased light sensitivity relative to the NinaE $E^{p p 100}$ mutant (Fig. 5A). Although these observations strongly suggested that arrestin contributed to the reduced light sensitivity of the NinaE $E^{p 100}$ mutant, Figures $4 A$ (bottom row) and $5 A$ revealed that the arr $2^{Y 20 S T O P} N i n a E^{p p 100}$ double mutant remained less sensitive to light relative to wild-type flies. This remaining desensitization in the $\operatorname{arr} 2^{Y 20 S T O P}$ NinaE $E^{P 100}$ fly may be attributable to either the NINAE ${ }^{\mathrm{Pp} 100}$ molecules being constitutively active or a reduced interaction between NINAE ${ }^{\mathrm{Pp} 100}$ and the heterotrimeric G-protein.

To investigate whether the NINAE ${ }^{\mathrm{p} 100}$ molecules were constitutively active and induced persistent opening of the lightactivated channels, we recorded whole-cell membrane currents to voltage steps in the dark (Fig. $5 C-F$ ). Wild-type cells revealed no significant currents under these conditions (Fig. 5C). However, small but significant (Yoon et al., 2000) currents were recorded from both Nina $E^{p p 100}$ and arr $2^{\text {Y20STOP }}$ NinaE $^{p{ }^{100}}$ mutant cells (Fig. 5D,E, respectively). The characteristics of these currents and their ability to be blocked by a low concentration of $\mathrm{La}^{3+}$ (Fig. $5 F$ ) suggested that they originate from constitutively open transient receptor potential (TRP) channels (Hardie and
Minke, 1994), which are the major light-activated channels (for review, see Minke and Hardie, 2000). These constitutive currents were recorded in $58 \%$ of the NinaE ${ }^{p p 100}$ cells and $74 \%$ of the arr $2^{\text {Y2OSTOP }}$ NinaE ${ }^{p p 100}$ double mutant photoreceptors (Fig. $5 B$ ). Because the arr $2^{Y 20 S T O P}$ mutant cells failed to exhibit any significant whole-cell membrane currents to voltage steps in the dark (data not shown), the arr $2^{Y 20 S T O P}$ mutation only contributed to the constitutive opening of the TRP channels in the presence of the NINAE ${ }^{\text {pp100 }}$ mutant rhodopsin (Fig. 5B). Although arrestin partially masked the production of the constitutive current, possibly through a stable NINAE ${ }^{\text {pp }}{ }^{100}-$ ARR2 complex, it is not clear why some of the mutant cells were not constitutively active. This may be related to variability in the level of rhodopsin among different NinaE $E^{p p 100}$ cells, which is consistent with the large variability in the light sensitivity between different NinaE ${ }^{p p 100} \mathrm{mu}-$ tant cells (Fig. 4B).

\section{The dominant NinaE ${ }^{p p 100}$ mutant requires both $\mathrm{G}_{\mathrm{q}} \alpha$ and ARR2 proteins for rapid degeneration}

It was shown previously that the norp $A, r d g B$, and $r d g C$ mutations produced a persistent NINAE-ARR2 complex that induced photoreceptor cell apoptosis (Alloway et al., 2000; Kiselev et al., 2000). Because the electrophysiology suggested that a stable NI$\mathrm{NAE}^{\mathrm{Pp} 100}-\mathrm{ARR} 2$ complex was present, we examined whether loss of the ARR2 protein suppressed the rapid NinaE $E^{p 100}$ dependent degeneration. At $10 \mathrm{~d}$ after eclosion, wild-type photoreceptors possessed large rhabdomeres (Fig. 6A), whereas a 4-dold NinaE $E^{p 100}$ mutant possessed significantly smaller and disorganized rhabdomeres and signs of photoreceptor cell death (Fig. 6D). The arr $2^{Y 2 O S T O P}$ null mutant possessed slightly smaller and irregular-shaped rhabdomeres relative to wild type (Fig. $6 B$ ), which is consistent with the light-dependent deep pseudopupil loss described previously (Alloway and Dolph, 1999). The 4-dold $\operatorname{arr} 2^{\text {Y2OSTOP }}$ NinaE ${ }^{\text {pp100 }}$ double mutant also exhibited small and abnormally shaped rhabdomeres (Fig. $6 E$ ), similar to the arr $2^{\text {Y2OSTOP }}$ mutant. However, neither arr2 ${ }^{\text {Y2OSTOP }}$ nor arr $2^{\text {Y20STOP }}$ NinaE ${ }^{\text {Pp100 }}$ exhibited the massive rhabdomere loss and photoreceptor cell death that occurred in the Nina $E^{p 100} \mathrm{mu}$ tant. This suggested that a stable NINAE ${ }^{\text {pp100}}-$ ARR2 complex induced both photoreceptor desensitization and cell death. Furthermore, the time course of deep pseudopupil loss revealed that the $\operatorname{arr} 2^{\text {Y2OSTOP }}$ mutation significantly slowed, but did not abolish, the NinaE ${ }^{p p 100}$ degeneration in both constant light and constant darkness (data not shown). Thus, the NINAE ${ }^{\text {pp100-ARR2 }}$ complex was not the sole cause of retinal degeneration, and an additional independent mechanism must participate in generating the Nina $E^{p p 100}$ mutant phenotypes.

Because the electrophysiology revealed that the NINAE ${ }^{\text {pp100 }}$ mutant protein was constitutively active, we examined whether activation of the light-sensitive channels induced the retinal degeneration phenotype. It was demonstrated previously that the early-onset retinal degeneration in the $r d g A^{B S 12}$ mutant, which possesses constitutively active light-sensitive channels, was significantly slowed by the $\operatorname{tr} p^{301}$ mutation (Raghu et al., 2000). We examined whether mutants blocking three different points in the phototransduction cascade could suppress the rapid NinaE $E^{p 100}$. dependent degeneration. The mutations we tested corresponded to the $d g q$ (which encodes the $\mathrm{G}_{\mathrm{q}} \alpha$ that is stimulated by lightactivated metarhodopsin), norpA (which encodes the phospholipase $\mathrm{C}$ that is activated by $\mathrm{G}_{\mathrm{q}} \alpha$ ), and $\operatorname{trp}$ genes (which encodes the major light-activated channel). The norp $A^{p 24}$ mutant exhibited no signs of retinal degeneration when raised for $6 \mathrm{~d}$ in constant darkness (Fig. 7B). In contrast, the NinaE $E^{p 100}$ mutant pos- 
sessed the previously described degeneration phenotype after $6 \mathrm{~d}$ in constant darkness (Fig. 7C). Although norp $A^{p 24}$; NinaE $E^{p 100} \mathrm{ex}$ hibited reduced and misshapen rhabdomeres after $6 \mathrm{~d}$ in constant darkness (Fig. $7 D$ ), it lacked the severe degeneration that was present in the NinaE $E^{p 100}$ mutant. This suggests that norp $A^{p 24}$ slightly delayed the NinaE $E^{p p 100}$ degeneration. Similarly, the $\operatorname{trp}^{301}$ mutation only slightly suppressed $\mathrm{Ni}$ $n a E^{p p 100}$ degeneration relative to the arr $2^{Y 20 S T O P}$ mutation (Fig. 7E). Thus, constitutive activation of the phototransduction cascade is only a minor component of the NinaE $E^{p 100}$ degeneration mechanism.

Surprisingly, a mutation in the $d g q$ gene, which encodes the $\mathrm{G}_{\mathrm{q}} \alpha$ subunit target for metarhodopsin (Lee et al., 1990, 1994; Scott et al., 1995), suppressed the Ni$n a E^{p p 100}$ deep pseudopupil loss substantially better than the $\operatorname{tr} p^{301}$ mutation (Figs. $6 \mathrm{~K}, 7 \mathrm{E}$, respectively). At $10 \mathrm{~d}$ after eclosion, the $d g q^{1}$ mutant possessed only a few missing rhabdomeres relative to the massive rhabdomere loss and photoreceptor cell death in NinaE $E^{p 100}$ (Fig. 6C,D, respectively). The ultrastructure of a 10-d-old $d g q^{1}$; NinaE $E^{p p^{100}}$ double mutant exhibited only minor photoreceptor abnormalities that were similar to the $d g q^{1}$ mutant (Fig. $6 F, C$, respectively). Furthermore, the time course of deep pseudopupil loss in the $d g q^{1}$; NinaE $E^{p p 100}$ double mutant was similar to the $d g q^{l}$ mutant in both dark and light-raised flies, which was significantly slower than the NinaE $E^{p 100}$ mutant (Fig. $6 \mathrm{~K})$. However, both the $d g q^{1}$; Nina $E^{p p 100}$ and $\operatorname{arr} 2^{\text {Y2OSTOP }}$ NinaE $E^{\text {p100 }}$ double mutants still exhibited a slow retinal degeneration (Fig. 6E,F,K). After $20 \mathrm{~d}$, the $d g q^{l}$; NinaE $E^{\text {pp1OO }}$ and arr2 ${ }^{\text {Y2OSTOP }}$ NinaE $E^{\text {pp100 }}$ double mutants exhibited massive photoreceptor cell death (Fig. $6 I, H$, respectively) relative to an equivalent aged wildtype retina (Fig. $6 J$ ). Surprisingly, the $d g q^{1}$;

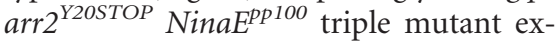
hibited only very minor rhabdomeric abnormalities after $40 \mathrm{~d}$ (Fig. 6G). The absence of significant cell death in this 40-dold triple mutant relative to either of the 20-d-old double mutants and the minor suppression of NinaE $E^{p 100}$ degeneration by the norp $A^{p 24}$ and $\operatorname{trp} p^{301}$ mutants revealed that both the ARR2 and $\mathrm{G}_{\mathrm{q}} \alpha$ proteins, but not activation of the light-sensitive channels, play major roles in the rapid NinaE $E^{p 100}$ dependent retinal degeneration.
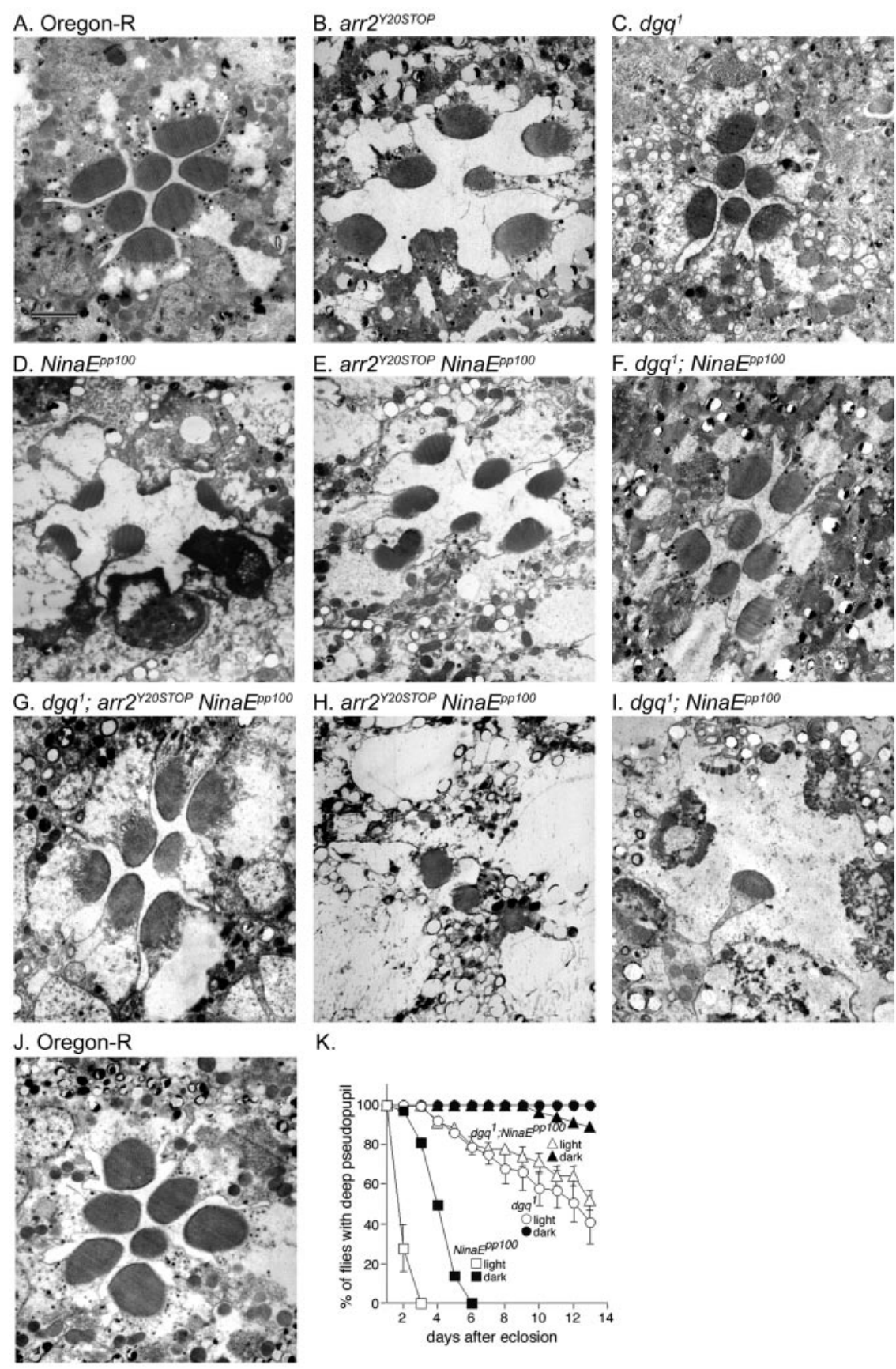

I. $d g q^{1} ; \operatorname{NinaE}^{p p 100}$

K.
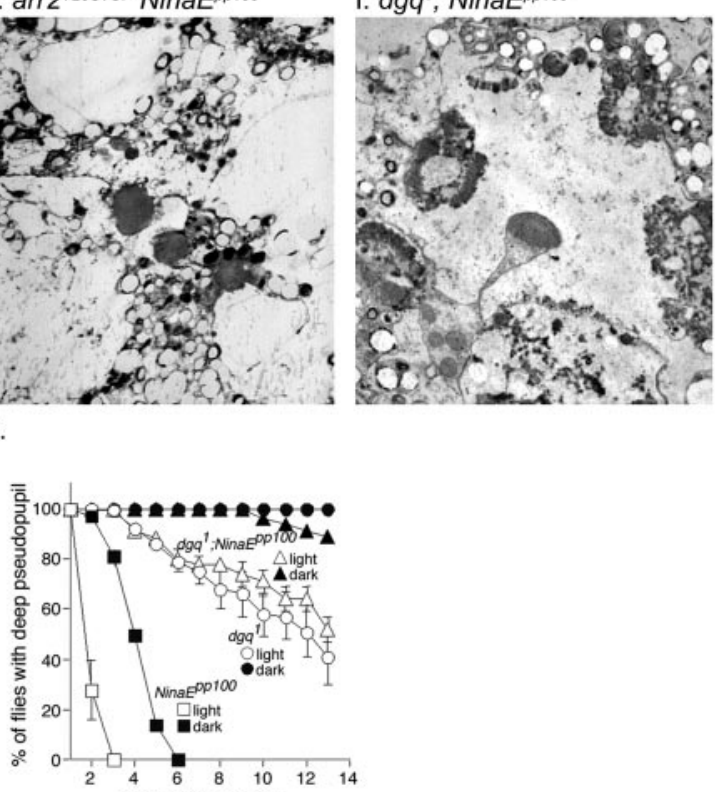

days after eclosion

Figure 6. The rapid Nina $E^{p p 100}$ degeneration requires both the $A R R 2$ and $G_{q} \alpha$ proteins. Wild-type (Oregon- $\left.R ; A, J\right)$, Nina $E^{p p 100}$

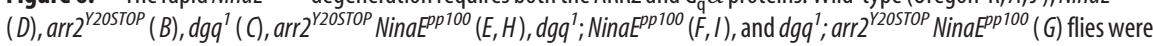
raised for $4 \mathrm{~d}(B, D, E), 10 \mathrm{~d}(A, C, F), 20 \mathrm{~d}(H, I)$, or $40 \mathrm{~d}(G, J)$ sectioned and examined by electron microscopy. Although both the $\operatorname{arr}^{\text {Y2OSTOP }}$ and $d g q^{7}$ mutants exhibit smaller, disorganized and occasional missing rhabdomeres relative to wild type, they are

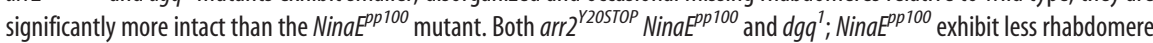

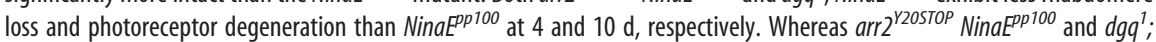
NinaE ${ }^{p 100}$ possess massive degeneration at $20 \mathrm{~d}$, the $\mathrm{dgq}^{1}$; arr2 $^{\mathrm{Y} 20 \text { STOP }}$ Nina $^{E^{p 100}}$ triple mutant exhibits only very minor abnormalities in the rhabdomere. Scale bar (in $A$ ): $A-J, 1 \mu \mathrm{m} . K$, The time course of deep pseudopupil loss of various genotypes [NinaE ${ }^{p p 100}$ flies (squares), $d g q^{7}$ flies (circles), and $d g q^{7}$; NinaE $E^{p 100}$ double mutants (triangles)] raised in either constant light or constant darkness (open and filled symbols, respectively). The percentages of flies retaining a deep pseudopupil on each day were plotted against their age, with each symbol representing the average from four independent trials of $100-150$ flies per trial.

\section{The dominant NINAE ${ }^{\mathrm{pp} 100}$ protein causes a constitutive disassociation and translocation of the heterotrimeric G-protein}

Whole-cell recordings revealed that the NinaE $E^{p 100}$ mutant photoreceptors were constitutively active (Fig. $5 B, D$ ), but the weak suppression of the NinaE $E^{p 100}$-dependent retinal degeneration by norpA or trp suggested that constitutive activation of the lightsensitive channels was not required for the rapid NinaE $E^{p 100}$. dependent retinal degeneration. We examined the extent of the constitutive activation by comparing the GTPase activity in wild- 

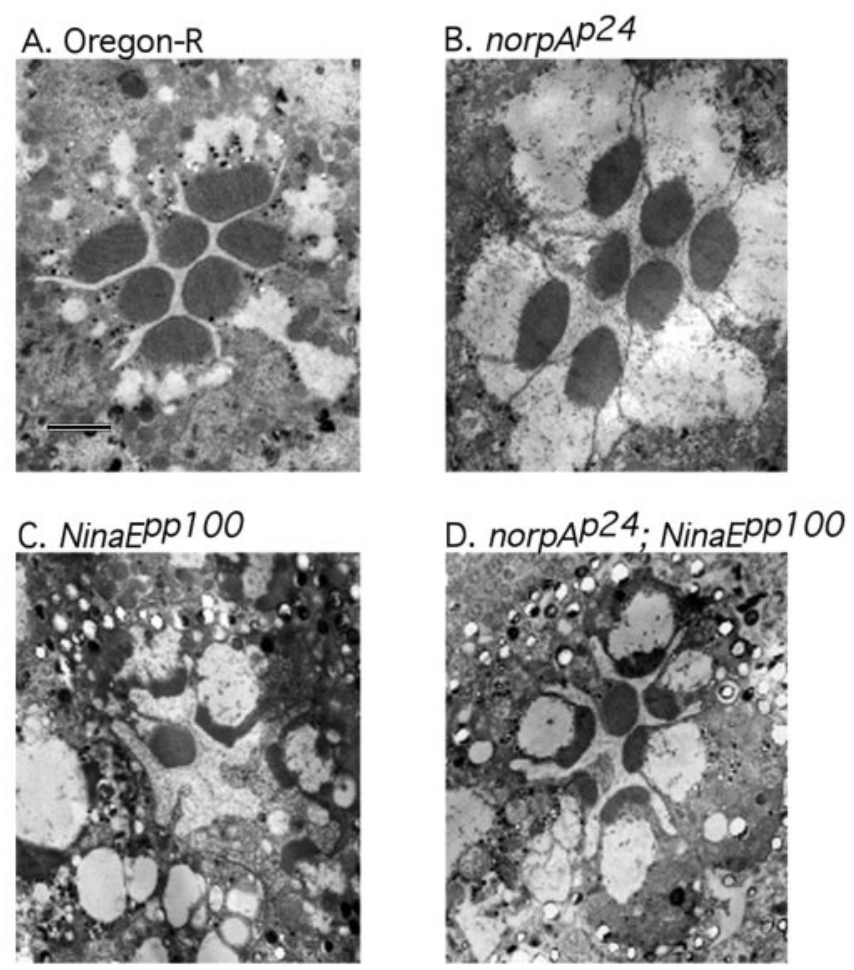

E.

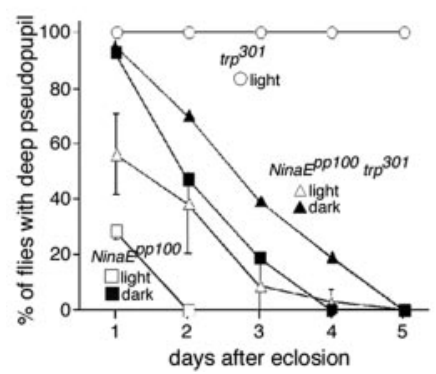

Figure 7. The norp $A^{p 24}$ and $t r p^{301}$ mutations only weakly suppress the rapid NinaEp $E^{p 00}$ retinal degeneration. Wild-type (Oregon-R; $A), \operatorname{norp}^{p 24}(B), \operatorname{Nina}^{p p 100}\left(C\right.$, and norpA $A^{p 24}$; $\operatorname{Nina}^{p p 100}(D)$ flies were raised for either $10 \mathrm{~d}(A)$ or $6 \mathrm{~d}(B-D)$, sectioned, and examined by electron microscopy. Although the norp $A^{p 24}$; Nina $E^{p p 100}$ double mutant exhibits rhabdomere shrinkage and disruption of the cell body relative to norp $A^{p 24}$, it lacks the massive cell degeneration characteristics observed in NinaE ${ }^{p p 100}$. Scale bar (in $A$ ): $A-D, 1 \mu \mathrm{m}$. E, The time course of deep pseudopupil loss of various genotypes [Nina $E^{p p 100}$ flies (squares), $\operatorname{trp}^{301}$ flies (circles), and Nina ${ }^{p p 100} \operatorname{trp}^{301}$ (triangles)] raised in either constant light or constant darkness (open and filled symbols, respectively). The percentages of flies retaining a deep pseudopupil on each day were plotted against their age, with each symbol representing the average from four independent trials of $100-150$ flies per trial.

type and Nina $E^{p p 100}$ mutant flies. Whereas we found high levels of light-dependent GTPase activity in wild-type flies, significantly lower light-dependent GTPase activity levels were measured in the NinaE $E^{p p 100}$ mutant (Fig. 8A), which suggested that a large fraction of the $G_{q} \alpha$-protein was not activated in the Nina $E^{p p 100}$ mutant. We demonstrated previously that the $\mathrm{G}_{\mathrm{q}} \alpha$-protein translocates from the rhabdomeric membrane to the cytosol during blue light illumination (Kosloff et al., 2003). Therefore, we examined whether the $\mathrm{G}_{\mathrm{q}} \alpha$-protein in the constitutively active Nina$E^{p p 100}$ mutant photoreceptors was predominantly in the cytosol. In wild-type flies, $\sim 80 \%$ of the $\mathrm{G}_{\mathrm{q}} \alpha$ was associated with the membrane fraction in the dark (Fig. $8 B$ ), which represented the GDPbound $\mathrm{G}_{\mathrm{q}} \alpha$ in the heterotrimer. During illumination, $\sim 70 \%$ of the $G_{\mathrm{q}} \alpha$ translocated into the cytosol (Fig. $8 B$ ). In contrast,
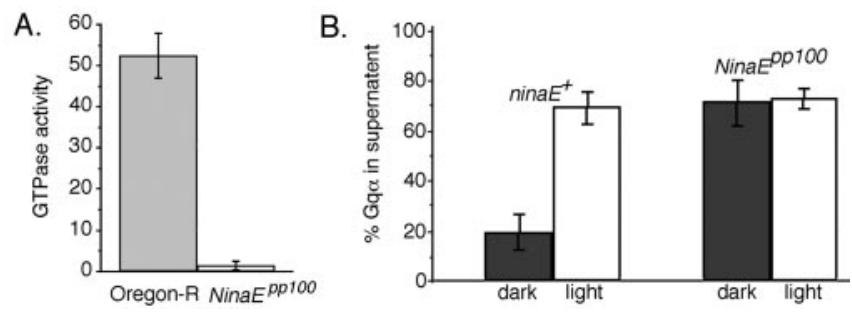

Figure 8. The subcellular distribution of the $\mathrm{G}_{\mathrm{q}} \alpha$-protein is altered in the NinaE ${ }^{\mathrm{pp}^{100}}$ mutant relative to wild type. $A$, The light-dependent GTPase activity (picomoles of phosphate per milligram of total protein per minute) was determined by subtracting the GTPase activity measured during orange light illumination from the GTPase activity measured during blue light illumination in the same experiment $(n=4)$. The histogram shows negligible blue lightstimulated GTPase activity in the NinaE ${ }^{p 100}$ mutant relative to wild-type flies. Furthermore, both the NinaE ${ }^{p p 100}$ mutant and wild-type flies exhibited the same low level of GTPase activity in orange light (data not shown). $B$, The subcellular distribution of the $\mathrm{G}_{\mathrm{q}} \alpha$-protein was examined in wild-type (ninaE $E^{+}$) and NinaE $E^{p p 100}$ mutant heads. The percentage of total $\mathrm{G}_{q} \alpha$-protein in the supernatant was determined in both light and dark conditions by subcellular fractionation and immunoblots. Although light treatment induced the translocation of $\mathrm{G}_{\mathrm{q}} \alpha$ to the cytosol in wild-type flies, light did not alter the percentage of $\mathrm{G}_{q} \alpha$-protein in the NinaE $E^{p p 100}$ supernatant $(n=4)$. Furthermore, the stable percentage of $\mathrm{G}_{\mathrm{q}} \alpha$ in the NinaE ${ }^{p p 100}$ supernatant was equivalent to the percentage of $\mathrm{G}_{\mathrm{q}} \alpha$ in the light-stimulated wild-type supernatant.

$\sim 70 \%$ of the $\mathrm{G}_{\mathrm{q}} \alpha$ was present in the cytosol of the NinaE $E^{p 100}$ mutant, regardless of illumination (Fig. $8 \mathrm{~B}$ ). This persistent and abnormal distribution of the $\mathrm{G}_{\mathrm{q}} \alpha$-protein in the cytosol could partially account for both the absence of light-dependent GTPase activity and the desensitization of the Nina $E^{p p 100}$ mutant photoreceptor cell.

\section{The formation of a stable rhodopsin-arrestin complex is required for the rapid photoreceptor degeneration} The ability of the arr ${ }^{\text {YZOSTOP }}$ mutation to significantly reduce the desensitization of the NinaE $E^{p 100}$ photoreceptor and suppress the Nina $E^{p p 100}$ degeneration suggested that the ARR2 protein persistently bound the NINAE ${ }^{\mathrm{pp} 100}$ mutant protein (Alloway et al., 2000; Kiselev et al., 2000). ARR2 is predominantly a soluble protein when rhodopsin is not photoactivated (such as during or after orange light illumination) (Fig. 9). However, during blue light conversion of rhodopsin to metarhodopsin, $\sim 80 \%$ of ARR2 protein binds metarhodopsin in the membrane (Fig. 9) (Byk et al., 1993). The ARR2 binding to the membrane is rhodopsin dependent, because only low levels of ARR2 are detected in $n i$ $n a E^{I 17}$ null mutant membrane fractions under either light condition (Fig. 9). In contrast, a significant percentage of ARR2 remained associated with the NinaE $E^{p 100}$ membrane, regardless of the light conditions (Fig. 9). The lower percentage of ARR2 in the newly enclosed NinaE ${ }^{p 100}$ membrane relative to the wild-type membrane after blue light illumination is consistent with the $\sim 80 \%$ reduction of NINAE protein in the NinaE $E^{p 100}$ mutant compared with wild type (Fig. $1 F$ ). Thus, the role of ARR2 in the rapid degeneration and strong desensitization in the NinaE $E^{p 100}$ mutant is through its persistent binding to $\operatorname{NINAE}^{\mathrm{pp} 100}$.

The NinaE $E^{p p 100}$ mutant degenerates through two independent pathways that involve the abnormal distribution of the ARR2 and $\mathrm{G}_{\mathrm{q}} \boldsymbol{\alpha}$

Because both the $d g q^{I}$ and $a r r 2^{\text {Y2OSTOP }}$ mutations significantly suppressed the rapid Nina $E^{p p 100}$-dependent retinal degeneration (Fig. 6), we examined whether the $\mathrm{G}_{\mathrm{q}} \alpha$-protein was required for the persistent association of $\mathrm{ARR} 2$ with the $\mathrm{NINAE}^{\mathrm{pp} 100}$ protein. We found that the percentage of ARR2 protein present in the membrane fraction of $d g q^{1}$; Nina $E^{p 100}$ double mutants was es- 
A.
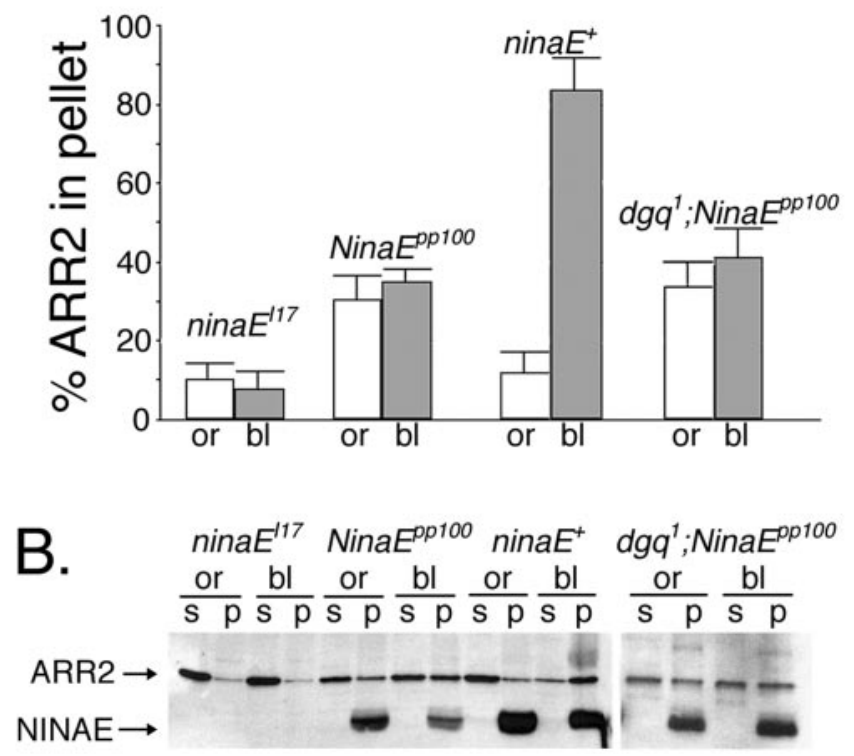

Figure 9. The ARR2 protein is persistently bound to the NINAE ${ }^{\mathrm{pp} 100}$ mutant rhodopsin. The subcellular distribution of the ARR2 protein was examined in wild-type ( $n i n a E^{+}$) and mutant (ninaE $E^{177}$,NinaE ${ }^{p p 100}$, and $d g q^{1}$; Nina $E^{p p 100}$ ) heads ( $<4 \mathrm{hr}$ after eclosion). $A$, The percentage of total ARR2 protein in the membrane pellet was determined using either 2 min of orange (or) or 2 min of blue (bl) light illumination, followed by subcellular fractionation and immunoblots $(n=5)$. The Nina $E^{p p 100}$ mutant had the same percentage of ARR2 protein in the pellet, regardless of the light conditions. This stable association was significantly more than that observed in the ninaE $E^{177}$ null mutant and significantly less than the wild-type flies under blue light illumination. $B, A$ representative immunoblot of the subcellular distribution of ARR2 and NINAE proteins in ninaE ${ }^{117}$, NinaE $E^{p p 100}$, ninaE ${ }^{+}$, and $d g q^{1}$; Nina $E^{p p 100}$ fly heads. The detection of NINAE in only the membrane pellet fraction demonstrates the completeness of removing the membrane from the soluble fractions.

sentially the same as in NinaE $E^{p 100}$ mutant flies (Fig. 9). Because the loss of the $G_{\alpha} \alpha$ protein did not reduce the level of the persistent NINAE ${ }^{\text {pp100 }}-$ ARR2 complexes, the $d g q^{1}$ suppression of $\mathrm{Ni}$ $n a E^{p p 100}$ degeneration must be arrestin independent. This demonstrates that $\mathrm{G}_{\mathrm{q}} \alpha$ is involved in a novel mechanism for the rapid Nina $E^{p p 100}$ degeneration, which may involve its predominant and persistent localization in the cytosol. Furthermore, the complete suppression observed in the 40 -d-old $d g q^{1}$; arr $2^{\text {Y2OSTOP }} \mathrm{Ni}$ $n a E^{p p 100}$ triple mutant clearly demonstrates that both the $\mathrm{G}_{\mathrm{q}} \alpha$ and ARR2-dependent degeneration pathways are both necessary and sufficient to account for the entire Nina $E^{p p^{100}}$-dependent degeneration process.

\section{Discussion}

The NinaE ${ }^{p p 100}$ mutant exhibits a novel retinal degeneration phenotype

We isolated a novel dominant rhodopsin mutation, NinaE $E^{p 100}$, that requires the presence of both $\mathrm{G}_{\mathrm{q}} \alpha$ and arrestin to induce a rapid degeneration. Transgenic expression of an in vitrogenerated glycine-to-arginine mutation at position 299 conclusively demonstrated that this is the molecular cause of the dominant Nina $E^{p 100}$ mutant phenotype (Fig. 2C). Previously, several dominant rhodopsin mutations were isolated that blocked rhodopsin trafficking and maturation out of the endoplasmic reticulum and slowly degenerated (Colley et al., 1995; Kurada and O’Tousa, 1995; Kurada et al., 1998). Several lines of data demonstrated that the NinaE $E^{p 100}$ mutation did not affect rhodopsin trafficking and maturation. First, the nina $E^{D 1}$ mutation, which is one of the most severe of the previously isolated dominant rhodopsin mutations (Kurada and O'Tousa, 1995; Kurada et al., 1998), suppressed the rapid NinaE ${ }^{p p 100}$ degeneration (Fig. 3). Therefore, the NINAE ${ }^{\text {pp100 }}$ protein must exert its effect subsequent to the ninaE $E^{D 1}$ trafficking defect. Second, NinaE $E^{p 100} / n i$ $n a E^{+}$flies lacked immature forms of rhodopsin on immunoblots (Fig. $1 F$ ). Thus, both wild-type and NINAE ${ }^{\text {pp100 }}$ mutant proteins must complete their maturation, although the NINAE ${ }^{\mathrm{pp} 100}$ protein was present at $\sim 20 \%$ of the $\operatorname{NINAE}^{+}$protein level (Fig. 1 F). Third, electron microscopy independently immunolocalized both epitope-tagged NINAE $^{+}$and epitope-tagged NINAE ${ }^{\text {pp100 }}$ in the rhabdomeres of $\mathrm{NinaE}^{\mathrm{pp} 100} /$ ninaE $^{+}$flies (data not shown), which confirmed that both forms reach the rhabdomere. Therefore, Nina $E^{p p^{100}}$ represents a new Drosophila model for dominant rhodopsin mutations.

\section{The mechanisms underlying the highly reduced sensitivity to light in the NinaE ${ }^{p p 100}$ mutant and its implications on congenital night blindness (CNB) in humans}

The strongly desensitized NinaE $E^{p 100}$ photoreceptor was characterized by the following: (1) the intense light required to elicit a small-amplitude response that lacked the typical bump noise (Fig. 4A), (2) the increased light intensity necessary to reach the saturated amplitude (Fig. $4 \mathrm{~B}$ ), and (3) the shift of the intensityresponse relationship toward higher light intensity levels (Figs. $4 B, 5 A)$. Although either the $80 \%$ decrease in the number of rhodopsin molecules (Fig. $1 F$ ) or the $80 \%$ decrease of $\mathrm{G}_{\mathrm{q}} \alpha$ protein associated with the membrane (Fig. $8 B$ ) would reduce the NinaE $E^{p 100}$ light sensitivity, they are insufficient to account for the observed four orders of magnitude reduction. A more significant mechanism is the constant state of light adaptation that results from the persistent NINAE ${ }^{\mathrm{pp} 100}-\mathrm{ARR} 2$ complex (Fig. 9). Normally, rhodopsin is present at a 5:1 molar ratio to ARR2 (Dolph et al., 1993). However, the NINAE ${ }^{\text {pp100 }}$ mutant protein is expressed at only $20 \%$ of the wild-type level of rhodopsin in wild-type photoreceptors (Fig. $1 F$ ), which yields an $\sim 1: 1$ molar ratio with ARR2. Thus, if $30-40 \%$ of the ARR2 is associated with the NinaE $E^{p 100}$ membrane, approximately an equivalent percentage of the NINAE ${ }^{\mathrm{Pp} 100}$ mutant protein is likely in a NINAE ${ }^{\text {Pp100 }}$ ARR2 complex. This would correspond to $\sim 35 \%$ of the NINA$\mathrm{E}^{\mathrm{pp} 100}$ being present in the persistent complex, which corresponds to $6-8 \%$ of the rhodopsin level in wild-type photoreceptors. This complex could account for the shift in the intensity-response curve toward brighter light (Fig. 5A). The fact that the light response in a fraction of mutant cells reached a normal peak amplitude at intense lights (Fig. $4 B$ ) is consistent with a significant number of light-sensitive rhodopsin molecules (i.e., $\sim 10^{3}-10^{5}$ ) (Johnson and Pak, 1986) not being bound by arrestin. The NINAE ${ }^{\text {Pp100}}-$ ARR2 complex would also account for the minimal light-stimulated GTPase activity in the NinaE $E^{p 100} \mathrm{mu}-$ tant photoreceptor cell. However, genetic elimination of ARR2 only partially increased the light sensitivity of the mutant Nina$E^{p p 100}$ (Figs. 4A, 5A), which could be explained by two different mechanisms. First, constitutively active NINAE ${ }^{\text {Pp100 }}$ would continually open the light-sensitive channels (Fig. 5), which leads to a persistent $\mathrm{Ca}^{2+}$ influx and photoreceptor desensitization. Second, activated $\mathrm{G}_{\mathrm{q}} \alpha$-protein may stimulate the internalization of specific G-protein-coupled receptors in the absence of arrestin binding (Rochdi and Parent, 2003). Thus, constitutively active NINAE ${ }^{\mathrm{pP} 100}$ would activate $\mathrm{G}_{\mathrm{q}} \alpha$, which could drive internalization of both wild-type and NINAE ${ }^{\text {pp100 }}$ rhodopsin molecules to desensitize the photoreceptor cell. 
This strong desensitization response, with only a minor effect on retinal degeneration, is similar to the human $\mathrm{CNB}$ phenotype (Dryja, 2000). One form of autosomal dominant CNB results from constitutively active forms of rod opsin (Jin et al., 2003). Three dominant rod opsin mutations were shown to cause a significant shift in the intensity-response relationship of transgenic Xenopus rods (Jin et al., 2003). The constitutive activity and desensitization observed in the NinaE ${ }^{p p 100}$ mutant suggests that the NINAE ${ }^{\mathrm{pP} 100}$ mutant rhodopsin may serve as an important model for elucidating the molecular details of one form of autosomal dominant CNB.

\section{A novel mechanism for dominant rhodopsin-mediated retinal degeneration}

The Nina $E^{p p^{100}}$ dominant mutation induces three different degeneration mechanisms. The first involves constitutive opening of the light-sensitive channels. The Drosophila $r d g A^{B S 12}$ and $\operatorname{trp}{ }^{P 365}$ mutations were shown to constitutively depolarize the photoreceptor cell and cause retinal degeneration (Raghu et al., 2000; Yoon et al., 2000). Furthermore, three different $\operatorname{trp}$ alleles (including $\operatorname{tr} p^{301}$ ) significantly slowed the rapid $r d g A^{B S 12}$ retinal degeneration, which confirmed that the $r d g A^{B S 12}$ degeneration was dependent on the constitutive opening of the light-activated channels (Raghu et al., 2000). However, the constitutive current observed in the NinaE $E^{p 100}$ mutant was approximately fivefold smaller than that found in the Drosophila $r d g A^{B S 12}$ and $\operatorname{trp}{ }^{P 365}$ mutants (Raghu et al., 2000; Yoon et al., 2000) and was not found in all of the cells (Fig. 5B). Furthermore, the weak suppression by the norp $A^{p 24}$ and $\operatorname{trp}{ }^{301}$ mutations on the rapid NinaE $E^{p 100}$ degeneration (Fig. $7 D, E$, respectively) confirmed that the constitutive opening of the light-sensitive channels is a minor Nina $E^{p 100}$ degeneration mechanism.

The second degeneration mechanism involves the formation of a stable NINAE ${ }^{\text {pp100 }}$-ARR2 complex. A variety of Drosophila mutations were shown previously to induce retinal degeneration by generating a stable NINAE-ARR2 complex that was endocytosed in a clathrin-dependent manner (Alloway et al., 2000; Kiselev et al., 2000). However, NinaE $E^{p 100}$ is the first Drosophila rhodopsin mutation that persistently targeted ARR2 to the membrane in all light conditions (Fig. 9). On the basis of the arr $2^{Y 20 S T O P}$ mutation suppressing the NinaE ${ }^{p p 100}$ degeneration (Fig. 6), the NINAE ${ }^{\text {Pp100 }}-$ ARR2 complex accounts for a major Nina $E^{p p 100}$ degeneration mechanism. The K296E opsin mutation in transgenic mice exhibited an analogous dominant photoreceptor degeneration phenotype (Robinson et al., 1992; Li et al., 1995; Rim and Oprian, 1995). Although the K296E mutant opsin constitutively activated the heterotrimeric G-protein in cell culture, it failed to constitutively stimulate the phototransduction cascade in the mouse photoreceptor because it was persistently phosphorylated and bound by arrestin. This stable rhodopsinarrestin complex may be a common stimulator of photoreceptor apoptosis for constitutively active rhodopsin mutations rather than persistent activation of the phototransduction cascade.

The third and novel Drosophila degeneration mechanism requires $\mathrm{G}_{\mathrm{q}} \alpha$ but not activation of the visual transduction cascade, because neither the norp $A^{p 24}$ nor $\operatorname{trp} p^{301}$ mutations suppressed $N i n a E^{p p 100}$ degeneration to the same extent as $d g q^{1}$ (Figs. 7, 6, respectively). Furthermore, the inability of the $d g q^{1}$ mutation to disrupt the stable NINAE ${ }^{\text {pp100}}-$ ARR2 complex (Fig. 9) and the consistent presence of $70 \%$ of the $\mathrm{G}_{\alpha} \alpha$-protein in the cytosolic fraction of both NinaE $E^{p 100}$ and arr $2^{\text {Y2OSTOP }}$ NinaE $^{\text {Pp100 }}$ mutants (Fig. $8 B$ and data not shown, respectively) demonstrated that the $\mathrm{G}_{\mathrm{q}} \alpha$ - and ARR2-dependent degeneration mechanisms were in- dependent. The light-independent localization of most of the $\mathrm{G}_{\mathrm{q}} \alpha$-protein to the NinaE $E^{p 100}$ cytosol is consistent with the constitutively active NINAE $^{\mathrm{pp} 100}$ stimulating $\mathrm{G}_{\mathrm{q}} \alpha$ dissociation from $\mathrm{G} \beta \gamma$ and the subsequent depalmitoylation of $\mathrm{G}_{\mathrm{q}} \alpha$ (Kosloff et al., 2003). The cytosolic localization and the markedly decreased light-dependent GTPase activity in the NinaE $E^{p 100}$ mutant (Fig. 8) suggests that the NINAE $^{\text {pp100 }}$ protein either diminishes the interaction between the cytosolic $\mathrm{G}_{\mathrm{q}} \alpha$-GTP with the membraneassociated norpA-encoded phospholipase C effector molecule, which also functions as a GTPase activating protein (Cook et al., 2000), or suppresses the reassociation of the cytosolic $G_{q} \alpha$ with the membrane. Because the loss of $\mathrm{G}_{\mathrm{q}} \alpha$-protein significantly slowed the NinaE $E^{p 100}$ degeneration, the persistent cytosolic localization of $\mathrm{G}_{\mathrm{q}} \alpha$ induces the degeneration rather than its absence from the membrane.

Whereas most previously characterized retinal degeneration models identified a single major pathway, the NinaE ${ }^{p p 100}$ mutant identified two major degeneration mechanisms, the stable NI$\mathrm{NAE}^{\mathrm{Pp} 100}-\mathrm{ARR} 2$ complex and the $\mathrm{G}_{\mathrm{q}} \alpha$-dependent pathway. The ability of ninaE $E^{D 1}$ to prevent the rapid NinaE $E^{p 100}$-dependent degeneration (Fig. 3) is consistent with the requirement of NINA$\mathrm{E}^{\mathrm{pp} 100}$ to reach the rhabdomere and interact with the ARR2 and $\mathrm{G}_{\mathrm{q}} \alpha$ proteins. Recently, knock-out mice revealed that lightinduced degeneration also involved two mechanisms (Hao et al., 2002), one requiring the transducin $\alpha$ subunit and the other involving photoactivated metarhodopsin and/or its bleaching intermediates (Hao et al., 2002). Thus, the NinaE ${ }^{p 100}$ mutant may reveal not only the complex interactions between constitutive activation and desensitization but also the multiplicity of mechanisms that may be involved in some of the rhodopsindependent degeneration mutations.

\section{References}

Alloway PG, Dolph PJ (1999) A role for the light-dependent phosphorylation of visual arrestin. Proc Natl Acad Sci USA 96:6072-6077.

Alloway PG, Howard L, Dolph PJ (2000) The formation of stable rhodopsin-arrestin complexes induces apoptosis and photoreceptor cell degeneration. Neuron 28:129-138.

Ashburner M (1989) Drosophila: a laboratory manual. Cold Spring Harbor, NY: Cold Spring Harbor Laboratory.

Blumenfeld A, Erusalimsky J, Heichal O, Selinger Z, Minke B (1985) Lightactivated guanosinetriphosphate in Musca eye membranes resembles the prolonged depolarizing afterpotential in photoreceptor cells. Proc Natl Acad Sci USA 82:7116-7121.

Byk T, Bar-Yaacov M, Doza YN, Minke B, Selinger Z (1993) Regulatory arrestin cycle secures the fidelity and maintenance of the fly photoreceptor cell. Proc Natl Acad Sci USA 90:1907-1911.

Colley NJ, Cassil JA, Baker EK, Zuker CS (1995) Defective intracellular transport is the molecular basis of rhodopsin-dependent dominant retinal degeneration. Proc Natl Acad Sci USA 92:3070-3074.

Cook B, Bar-Yaacov M, Cohen Ben-Ami H, Goldstein RE, Paroush Z, Selinger Z, Minke B (2000) Phospholipase C and termination of G-protein-mediated signaling in vivo. Nat Cell Biol 2:296-301.

Dolph PJ, Ranganathan R, Colley NJ, Hardy RW, Socolich M, Zuker CS (1993) Arrestin functions in inactivation of G protein-coupled receptor rhodopsin in vivo. Science 260:1910-1915.

Dryja TP (2000) Molecular genetics of Oguchi disease, fundus albipunctatus, and other forms of stationary night blindness: LVII Edward Jackson Memorial Lecture. Am J Ophthalmol 130:547-563.

Dryja TP, Berson EL, Rao VR, Oprian DD (1993) Heterozygous missense mutation in the rhodopsin gene as a cause of congenital stationary night blindness. Nat Genet 4:280-282.

Franceschini N (1972) Pupil and pseudopupil in the compound eye of Drosophila. In: Information processing in the visual systems of arthropods (Wehner R, ed), pp 75-82. Berlin: Springer.

Gal A, Apfelstedt-Sylla E, Janecke AR, Zrenner Z (1997) Rhodopsin mutations in inherited dystrophies and dysfunctions. Prog Ret Eye Res 16:51-79.

Hao W, Wenzel A, Obin MS, Chen CK, Brill E, Krasnoperova NV, Eversole- 
Cire P, Kleyner Y, Taylor A, Simon MI, Grimm C, Reme CE, Lem J (2002) Evidence for two apoptotic pathways in light-dependent retinal degeneration. Nat Genet 32:254-260.

Hardie RC (1991) Voltage-sensitive potassium channels in Drosophila photoreceptors. J Neurosci 11:3079-3095.

Hardie RC, Minke B (1992) The trp gene is essential for a light-activated $\mathrm{Ca}^{2+}$ channel in Drosophila photoreceptors. Neuron 8:643-651.

Hardie RC, Minke B (1994) Spontaneous activation of light-sensitive channels in Drosophila photoreceptors. J Gen Physiol 103:389-407.

Hillman P, Hochstein S, Minke B (1983) Transduction in invertebrate photoreceptors: role of pigment bistability. Physiol Rev 63:668-772.

Jin S, Cornwall MC, Oprian DD (2003) Opsin activation as a cause of congenital night blindness. Nat Neurosci 6:731-735.

Johnson EC, Pak WL (1986) Electrophysiological study of Drosophila rhodopsin mutants. J Gen Physiol 88:651-673.

Kaushal S, Khorana HG (1994) Structure and function in rhodopsin. 7. Point mutations associated with autosomal dominant retinitis pigmentosa. Biochemistry 33:6121-6128.

Kiselev A, Subramaniam S (1994) Activation and regeneration of rhodopsin in the insect visual cycle. Science 266:1369-1373.

Kiselev A, Socolich M, Vinos J, Hardy RW, Zuker CS, Ranganathan R (2000) A molecular pathway for light-dependent photoreceptor apoptosis in Drosophila. Neuron 28:139-152.

Kosloff M, Elia N, Joel-Almagor T, Timberg R, Zars TD, Hyde DR, Minke B, Selinger Z (2003) Regulation of light-dependent Gqalpha translocation and morphological changes in fly photoreceptors. EMBO J 22:459-468.

Kurada P, O’Tousa JE (1995) Retinal degeneration caused by dominant rhodopsin mutations in Drosophila. Neuron 14:571-579.

Kurada P, Tonini TD, Serikaku MA, Piccini JP, O’Tousa JE (1998) Rhodopsin maturation antagonized by dominant rhodopsin mutants. Vis Neurosci 15:693-700.

Lee Y-J, Dobbs MB, Verardi ML, Hyde DR (1990) dgq: a Drosophila gene encoding a visual system-specific G alpha molecule. Neuron 5:889-898.

Lee Y-J, Shah S, Suzuki E, Zars T, O’Day PM, Hyde DR (1994) The Drosophila $d g q$ gene encodes a $\mathrm{G}$ alpha protein that mediates phototransduction. Neuron 13:1143-1157.

Leonard DS, Bowman VD, Ready DF, Pak WL (1992) Degeneration of photoreceptors in rhodopsin mutants of Drosophila. J Neurobiol 23:605-626.

Lewis EB, Bacher F (1968) Method of feeding ethyl methane sulfonate (EMS) to Drosophila males. Dros Inf Serv 43:193.

Li T, Franson W, Gordon JW, Berson EL, Dryja TP (1995) Constitutive activation of phototransduction is not a cause of photoreceptor degeneration. Proc Natl Acad Sci USA 92:3551-3555.

Milligan S, Alb JG, Elagina RB, Bankaitis VA, Hyde DR (1997) The phosphatidylinositol transfer protein domain of Drosophila Retinal Degeneration B protein is essential for photoreceptor cell survival and recovery from light stimulation. J Cell Biol 139:351-363.

Min KC, Zvyaga TA, Cypess AM, Sakmar TP (1993) Characterization of mutant rhodopsins responsible for autosomal dominant retinitis pigmentosa. Mutations on the cytoplasmic surface affect transducin activation. J Biol Chem 268:9400-9404.

Minke B, Hardie RC (2000) Genetic dissection of Drosophila phototransduction. In: Molecular mechanisms in visual transduction (Stavenga DG, van der Hope DJN, Pugh E, eds), pp 449-525. North Holland, The Netherlands: Elsevier.

O’Tousa JE, Baehr W, Martin RL, Hirsh J, Pak WL, Applebury ML (1985) The Drosophila ninaE gene encodes an opsin. Cell 40:839-850.

Paetkau DW, Elagin VA, Sendi LM, Hyde DR (1999) Isolation and characterization of Drosophila retinal degeneration B suppressors. Genetics 151:713-724.

Peretz A, Suss-Toby E, Rom-Glas A, Arnon A, Payne R, Minke B (1994) The light response of Drosophila photoreceptors is accompanied by an increase in cellular calcium: effects of specific mutations. Neuron 6:1257-1267.

Phelan JK, Bok D (2000) A brief review of retinitis pigmentosa and the identified retinitis pigmentosa genes. Mol Vis 6:116-124.

Raghu P, Usher K, Jonas S, Chyb S, Polyanovsky A, Hardie RC (2000) Constitutive activity of the light-sensitive channels TRP and TRPL in the Drosophila diacylglycerol kinase mutant, $r d g A$. Neuron 26:169-179.

Rao VR, Cohen GB, Oprian DD (1994) Rhodopsin mutation G90D and a molecular mechanism for congenital night blindness. Nature 367:639-642.

Rattner A, Sun H, Nathans J (1999) Molecular genetics of human retinal disease. Annu Rev Genet 33:89-131.

Rim J, Oprian DD (1995) Constitutive activation of opsin: interaction of mutants with rhodopsin kinase and arrestin. Biochemistry 34:11938-11945.

Robinson PR, Cohen GB, Zhukovsky EA, Oprian DD (1992) Constitutively active mutants of rhodopsin. Neuron 9:719-725.

Rochdi MD, Parent JL (2003) G $\alpha$ q-coupled receptor internalization specifically induced by $\mathrm{G} \alpha \mathrm{q}$ signaling. J Biol Chem 278:17827-17837.

Roof DJ, Adamian M, Hayes A (1994) Rhodopsin accumulation at abnormal sites in retinas of mice with a human $\mathrm{P} 23 \mathrm{H}$ rhodopsin transgene. Invest Ophtalmol Vis Sci 35:4049-4061.

Scott K, Becker A, Sun Y, Hardy R, Zuker CS (1995) Gq alpha protein function in vivo: genetic dissection of its role in photoreceptor cell physiology. Neuron 15:919-927.

Shetty KM, Kurada P, O’Tousa JE (1998) Rab6 regulation of rhodopsin transport. J Biol Chem 273:20425-20430.

Spradling AC (1986) P element-mediated transformation. In: Drosophila a practical approach (Roberts DB, ed), pp 175-196. Oxford: IRL.

Sung C-H, Makino C, Baylor D, Nathans J (1994) A rhodopsin gene mutation responsible for autosomal dominant retinitis pigmentosa results in a protein that is defective in localization to the photoreceptor outer segment. J Neurosci 14:5818-5833.

Vihtelic TS, Hyde DR, O'Tousa JE (1991) Isolation and characterization of the Drosophila retinal degeneration B ( $r d g B)$ gene. Genetics 127:761-768.

Vihtelic TS, Goebl M, Milligan S, O’Tousa JE, Hyde DR (1993) Localization of Drosophila retinal degeneration B, a membrane-associated phosphatidylinositol transfer protein. J Cell Biol 122:1013-1022.

Wang Q, Chen Q, Zhao K, Wang L, Traboulsi EI (2001) Update on the molecular genetics of retinitis pigmentosa. Ophthalmic Genet 22:133-154.

Yoon J, Ben-Ami HC, Hong YS, Park S, Strong LLR, Bowman J, Geng C, Baek K, Minke B, Pak WL (2000) Novel mechanism of massive photoreceptor degeneration caused by mutation in the trp gene of Drosophila. J Neurosci 20:649-659.

Zuker CS, Cowman AF, Rubin GM (1985) Isolation and structure of a rhodopsin gene from D. melanogaster. Cell 40:851-858. 